\title{
High-frequency limit of the Maxwell-Landau-Lifshitz system in the diffractive optics regime
}

\author{
LU Yong*
}

\begin{abstract}
We study semilinear Maxwell-Landau-Lifshitz systems in one space dimension. For highly oscillatory and prepared initial data, we construct WKB approximate solutions over long times $O(1 / \varepsilon)$. The leading terms of the WKB solutions solve cubic Schrödinger equations. We show that the nonlinear normal form method of Joly, Métivier and Rauch 4 applies to this context. This implies that the Schrödinger approximation stays close to the exact solution of MaxwellLandau-Lifshitz over its existence time. In the context of Maxwell-Landau-Lifshitz, this extends the analysis of Colin and Lannes [2] from times $O(|\ln \varepsilon|)$ up to $O(1 / \varepsilon)$.
\end{abstract}

\section{Contents}

1 Introduction 2

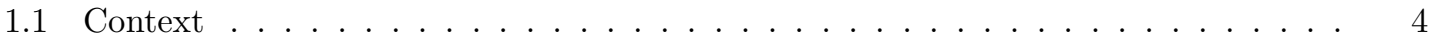

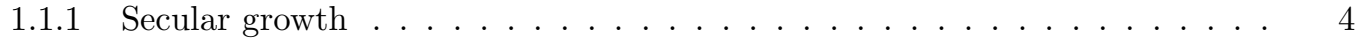

1.1.2 Maxwell-Bloch structure . . . . . . . . . . . . . . . . . . 6

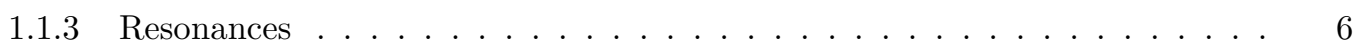

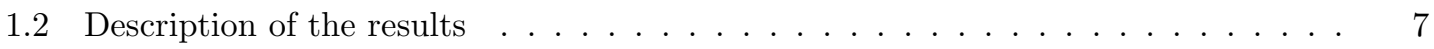

2 WKB expansion and approximate profile 9

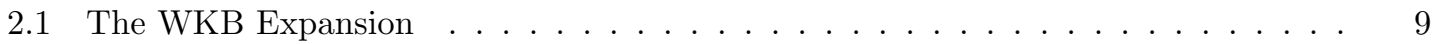

2.2 The approximate solution and the remainder $\ldots \ldots \ldots \ldots$

3 Maxwell-Bloch structure and long time existence. 18

3.1 Spectral decomposition . . . . . . . . . . . . . . . . . . 18

3.2 Strong transparency and normal form reduction . . . . . . . . . . . . . . . 19

4 Error estimates 22

$4.1 \quad$ First error estimate . . . . . . . . . . . . . . . . . 23

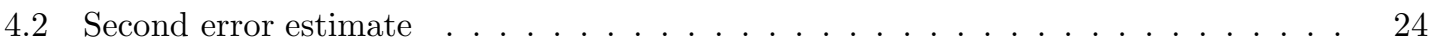

4.3 Proof of Theorem $1.3 \ldots \ldots \ldots \ldots \ldots \ldots \ldots$

${ }^{*}$ Université Paris-Diderot (Paris 7), Institut de Mathématiques de Jussieu, UMR CNRS 7586; e-mail: luyong@math.jussieu.fr 


\section{Introduction}

The state of a ferromagnet is described by the magnetization vector $M$. The evolution of $M$ with time $t$ without damping is governed by the Landau-Lifshitz equation

$$
\partial_{t} M=-M \times H
$$

where $H$ is the magnetic field. The electromagnetic field variables obey Maxwell equations

$$
\left\{\begin{array}{l}
\partial_{t} E-\nabla \times H=0 \\
\partial_{t} H+\nabla \times E=-\partial_{t} M
\end{array}\right.
$$

The Maxwell-Landau-Lifshitz system that we study is (1.1)-(1.2). The physical constants have been set equal to one. The spatial domain is $\mathbb{R}^{3}$. Observe that the Maxwell-Landau-Lifshitz system admits a family of constant solutions:

$$
(E, H, M)_{\alpha}=\left(0, \alpha M_{0}, M_{0}\right),
$$

for any $\alpha>0$ and any $M_{0} \in \mathbb{R}^{3}$. We are interested in the solutions that can be written as small, slowly variable perturbations of such constant solutions. The perturbations are measured in terms of an arbitrarily small positive parameter $\varepsilon$. We postulate the following form for the perturbations:

$$
E(t, x)=\varepsilon \tilde{E}(\varepsilon t, \varepsilon x), H(t, x)=\alpha M_{0}+\varepsilon \tilde{H}(\varepsilon t, \varepsilon x), M(t, x)=M_{0}+\varepsilon \tilde{M}(\varepsilon t, \varepsilon x) .
$$

If $(E, H, M)$ is a solution of (1.1)-(1.2), then $(\tilde{E}(t, x), \tilde{H}(t, x), \tilde{M}(t, x))$ satisfies the following system

$$
\left\{\begin{array}{l}
\partial_{t} \tilde{E}-\nabla \times \tilde{H}=0 \\
\partial_{t} \tilde{H}+\nabla \times \tilde{E}=-\partial_{t} \tilde{M} \\
\partial_{t} \tilde{M}=-\varepsilon^{-1} M_{0} \times \tilde{H}+\varepsilon^{-1} \alpha M_{0} \times \tilde{M}-\tilde{M} \times \tilde{H} .
\end{array}\right.
$$

Introduce the perturbation unknown $u=\left(\tilde{E}, \tilde{H}, \alpha^{\frac{1}{2}} \tilde{M}\right):[0, \infty) \times \mathbb{R}^{3} \rightarrow \mathbb{R}^{9}=\mathbb{R}^{3} \times \mathbb{R}^{3} \times \mathbb{R}^{3}$. Then the system (1.3) can be written as a symmetric quadratic hyperbolic system in $u$ :

$$
\partial_{t} u+A\left(\partial_{x}\right) u+\frac{1}{\varepsilon} L_{0} u=B(u, u),
$$

where $A$ and $L_{0}$ are defined by

$$
A\left(\partial_{x}\right)=\left(\begin{array}{ccc}
0 & -\partial_{x} \times & 0 \\
\partial_{x} \times & 0 & 0 \\
0 & 0 & 0
\end{array}\right), \quad L_{0}=\left(\begin{array}{ccc}
0 & 0 & 0 \\
0 & -M_{0} \times & \alpha^{\frac{1}{2}} M_{0} \times \\
0 & \alpha^{\frac{1}{2}} M_{0} \times & -\alpha M_{0} \times
\end{array}\right) .
$$

The bilinear form $B(\cdot, \cdot)$ is defined for any $u=\left(u^{1}, u^{2}, u^{3}\right), v=\left(v^{1}, v^{2}, v^{3}\right) \in \mathbb{R}^{3} \times \mathbb{R}^{3} \times \mathbb{R}^{3}$ by

$$
B(u, v)=\frac{1}{2}\left(\begin{array}{c}
0 \\
\alpha^{-\frac{1}{2}}\left(u^{3} \times v^{2}+v^{3} \times u^{2}\right) \\
-\left(u^{3} \times v^{2}+v^{3} \times u^{2}\right)
\end{array}\right) .
$$

It is easy to check that $A\left(\partial_{x}\right)=\sum_{j=1}^{3} A_{j} \partial_{j}$ with $A_{j}$ symmetric and that $L_{0}$ is skew-symmetric. We set $\alpha=1$ and $M_{0}=(1,0,0)$. 
In [7, Leblond did formal computations for Maxwell-Landau-Lifshitz system, and derived a DaveyStewartson system in the high-frequency limit, for diffractive times. Our goal is to justify the Davey-Stewartson approximation over long time interval of order $O(1 / \varepsilon)$. For technical reasons that are made clear in Section 1.1.3 and have to do with the issue of localizing the resonances, so we restrict our problem to one spatial dimension, where the Davey-Stewartson system degenerates into cubic Schrödinger equation. In one spatial dimension, the equation (1.4) becomes

$$
\partial_{t} v+A\left(e_{1}\right) \partial_{y} v+\frac{L_{0} v}{\varepsilon}=B(v, v),
$$

where the space variable is $y \in \mathbb{R}^{1}$, and the vector $e_{1} \in \mathbb{R}^{3}$ is

$$
e_{1}=(1,0,0)^{t} \text {. }
$$

We consider highly oscillatory initial data of the form

$$
v(0, y)=a(y) e^{i k y / \varepsilon}+\overline{a(y)} e^{-i k y / \varepsilon}+\varepsilon a_{1}(y, k y / \varepsilon)+\varepsilon^{2} a_{2}(y, k y / \varepsilon),
$$

where $a_{1}(y, \theta)$ and $a_{2}(y, \theta)$ are real-valued and $2 \pi$-periodic in $\theta$.

The system (1.7) is semilinear symmetric hyperbolic. For fixed $\varepsilon>0$, the local existence, uniqueness and regularity of local-in-time solutions to (1.7)-(1.9) in smooth Sobolev spaces are classical.

Our goal here is to describe the solutions in the high-frequency limit $\varepsilon \rightarrow 0$ over long times $O(1 / \varepsilon)$. There are three main issues.

(1). First, because of the fast oscillations, the Sobolev norm of the initial datum is unbounded as $\varepsilon \rightarrow 0$ : for any $s>0$,

$$
|v(0)|_{H^{s}}=\left|a(y) e^{i k y / \varepsilon}+\ldots\right|_{H^{s}}=O\left(\varepsilon^{-s}\right) \rightarrow \infty .
$$

By the classical theory of Cauchy problem of symmetric hyperbolic system, the existence time $t^{*}(\varepsilon)$ shrinks to 0 as $\varepsilon \rightarrow 0$.

We can go around this issue and show that the natural existence time is $O(1)$ by introducing profiles as in [3] and [4]. This means we consider a representation of the solutions by maps depending periodically on the fast variable $\theta \in \mathbb{T}=\mathbb{R} / 2 \pi \mathbb{Z}$ :

$$
v(t, y)=\left.V(t, y, \theta)\right|_{\theta=\frac{(-\omega t+k y)}{\varepsilon}},
$$

where $\omega \in \mathbb{R}$ is chosen such that the couple $(\omega, k)$ satisfies dispersion relation det $\left(-i \omega+A\left(e_{1}\right) i k+\right.$ $\left.L_{0}\right)=0$. Then to solve the Cauchy problem (1.7)-(1.9), it is sufficient to solve the following Cauchy problem in $V$ :

$$
\left\{\begin{array}{l}
\partial_{t} V+A\left(e_{1}\right) \partial_{y} V+\frac{1}{\varepsilon}\left\{-\omega \partial_{\theta}+A\left(e_{1}\right) k \partial_{\theta}+L_{0}\right\} V=B(V, V), \\
V(0, y, \theta)=a(y) e^{i \theta}+\overline{a(y)} e^{-i \theta}+\varepsilon a_{1}(y, \theta)+\varepsilon^{2} a_{2}(y, \theta) .
\end{array}\right.
$$

This is a symmetric hyperbolic system, for which the classical theory gives local-in-time wellposedness, uniformly in $\varepsilon$. Indeed, the data are now uniformly bounded with respect to $\varepsilon$ in Sobolev spaces with non-negative indices, the $L^{2}$ estimate is uniform in $\varepsilon$ in spite of the large $1 / \varepsilon$ prefactor because the operator $-\omega \partial_{\theta}+A\left(e_{1}\right) k \partial_{\theta}+L_{0}$ is skew-adjoint, and the commutator estimates are trivial because the operator $-\omega \partial_{\theta}+A\left(e_{1}\right) k \partial_{\theta}+L_{0}$ has constant coefficients.

(2). Second, the initial datum (1.9) has a large amplitude $O(1)$. In this regime, the maximal existence time a priori is $O(1)$, not $O(1 / \varepsilon)$. For (1.11), the classical $H^{s}(\mathbb{R} \times \mathbb{T})$ energy estimate for semilinear symmetric hyperbolic operators is

$$
\left.V(t)\right|_{H^{s}} \leq|V(0)|_{H^{s}}+C \int_{0}^{t}\left|V\left(t^{\prime}\right)\right|_{H^{s}} d t^{\prime}
$$


where the constant $C$ depends on $|V|_{L^{\infty}}$, implying, by Gronwall's lemma, the bound

$$
|V(t)|_{H^{s}} \leq|V(0)|_{H^{s}} e^{C t} .
$$

Over long times $O(1 / \varepsilon)$, the upper bound diverges to infinity as $\varepsilon \rightarrow 0$. We will overcome this problem by normal form reduction and a rescalling method, following Joly, Métivier and Rauch in [4 and Texier in [9]. This is in contrast to the situation in which the initial data have a small amplitude $O(\varepsilon)$, where the regime is said to be weakly nonlinear and the natural existence time is $O(1 / \varepsilon)$.

(3). Third, we are confronted with the issue of deriving uniform bounds for the corrector terms of the ansatz. If we denote $V=V^{0}+\varepsilon V^{1}+\ldots$ the WKB expansion, we want indeed to guarantee that $\varepsilon V^{1}$ is $o(1)$ in the time interval under consideration, otherwise the significance of the WKB expansion as an asymptotic expansion breaks down. However there typically arises an equation of the form $\left(\partial_{t}+\rho \partial_{y}\right) a=b$, where $a$ is a component of $V^{1}$ and $b$ depends on the leading term $V^{0}$.

For the trivial datum $a(0, y)=0$, the solution is $a(t, y)=\int_{0}^{t} b(t-s, y-\rho s) d s$, giving typically the estimate $\|a(t)\|_{H^{s}} \leq t\|b\|_{L^{\infty}\left(H^{s}\right)}$ and nothing better. Over long time $O(1 / \varepsilon)$, the upper bound is of order $O(1 / \varepsilon)$ implying that $\varepsilon V^{1}$ could well be $O(1)$. This phenomenon is called secular growth and was extensively studied by Lannes in [6].

For Maxwell-Landau-Lifshitz, we overcome the issues (2) and (3), and show existence up to times of order $O(1 / \varepsilon)$. We also show that the solution can be well approximated by WKB solutions, the leading terms of which satisfy cubic Schrödinger equations.

In the rest of this introduction, we describe in greater detail the issues associated with (2)-(3) above in upcoming Section 1.1, before turning to the statement of our results (in Section 1.2).

\subsection{Context}

\subsubsection{Secular growth}

In [2], Colin and Lannes study systems of the form (1.4) with highly oscillatory initial data

$$
u(0, x)=a(x) e^{i \vec{k} \cdot x / \varepsilon}+\overline{a(x)} e^{-i \vec{k} \cdot x / \varepsilon}+\varepsilon a_{1}\left(x, \frac{\vec{k} \cdot x}{\varepsilon}\right)+\varepsilon^{2} a_{2}\left(x, \frac{\vec{k} \cdot x}{\varepsilon}\right)+\ldots
$$

corresponding to (1.9) in several space dimensions.

They first show, under a weak transparency assumption (see Assumption 2.1 in [4]) ensuring that a WKB cascade exists that an a three-scale approximate profile of the form

$$
V^{a}(t, x, \theta)=\left.\sum_{j=0}^{2} \varepsilon^{j} V_{j}(\tau, t, x, \theta)\right|_{\tau=\varepsilon t}, \quad V_{j}(\tau, t, x, \theta)=\sum_{p} \varepsilon^{i p \theta} V_{j p}(\tau, t, x)
$$

can be constructed by three-scale WKB expansion over long times of order $O(1 / \varepsilon)$. The leading terms of the approximate solution solve Davey-Stewartson systems. Under the additional assumption that the Davey-Stewartson systems are well-posed, this proves existence of WKB solutions over time $O(1 / \varepsilon)$. As mentioned in Section 1.1, Colin and Lannes have to deal with the issue of secular growth. There arises in their analysis an equation of the form

$$
\left(\partial_{t}+\omega^{\prime}(k) \cdot \partial_{x}\right) \pi_{1} V_{11}=2 \pi_{1} B\left(V_{01},\left(\pi_{0} V_{10}\right)^{*}\right),
$$


where $\pi_{0}$ is a spectral projector (see (2.11) $)$ and $\left(\pi_{0} V_{10}\right)^{*}$ is from the following decomposition

$$
\pi_{0} V_{10}=\left(\pi_{0} V_{10}\right)^{*}+\left\langle\pi_{0} V_{10}\right\rangle
$$

where the part $\left\langle\pi_{0} V_{10}\right\rangle$ satisfies the transport equations $\left(\partial_{t}+\omega^{\prime}(k) \cdot \partial_{x}\right)\left\langle\pi_{0} V_{10}\right\rangle=0$ and the residual $\left(\pi_{0} V_{10}\right)^{*}$ satisfies the following homogeneous hyperbolic equation

$$
\left(\partial_{t}+\pi_{0} A\left(\partial_{x}\right) \pi_{0}\right)\left(\pi_{0} V_{10}\right)^{*}=0 .
$$

The couple $\left(V_{01},\left\langle\pi_{0} V_{10}\right\rangle\right)$ is the solution of a Davey-Stewartson system and is uniformly bounded in a Sobolev space over time interval $[0, T]_{\tau} \times \mathbb{R}_{t}$, where the vector $\left\langle\pi_{0} V_{10}\right\rangle$ is determined by the leading term $V_{01}$.

Given any initial datum to $\left(\pi_{0} V_{10}\right)^{*}$, the equation (1.14) implies a global solution $\left(\pi_{0} V_{10}\right)^{*}$. Back to (1.13), one may only generally have an estimate $\pi_{1} V_{11}=O(t)$, unbounded as $t \rightarrow \infty$. Assuming that the initial datum $a \in \Sigma^{s}=\left\{u \in H^{s+n^{+} / 2},(1+|\cdot|)^{s} \hat{u} \in L^{\infty}\right\}$ for some $s>3+n / 2$, Colin and Lannes prove a better secular growth estimate in $O(\ln t)$ for $\pi_{1} V_{11}$ (this is Proposition 2.3 in [2]). Then, assuming that the initial perturbation $\left(v-v^{a}\right)(0)$ is $O\left(\varepsilon^{2}\right)$, Colin and Lannes prove stability of the WKB solution in times $O(|\ln \varepsilon|$ ) (this is Proposition 3.1 in [2]).

Here, we consider the problem in one space dimension, with initial data in classical Sobolev spaces. We allow initial perturbations of size $O(\varepsilon)$, and give two results (see Theorem 1.3 below for a precise statement), the proofs of which rely heavily on the specific structure of the Maxwell-Landau-Lifshitz system.

First, assuming only the standard polarization condition for the leading amplitude $a$ in the initial datum (1.9), that is $a(y) \in \operatorname{ker}\left(-i \omega+A\left(e_{1}\right) i k+L_{0}\right)$, we give a stability result in intermediate times. This is statement ii in Theorem 1.4 below. We show that a WKB approximate solution can be constructed, of which the leading term solves cubic Schrödinger equation. We choose $\left(\pi_{0} V_{10}\right)^{*}(0)=0$ to eliminate the secular growth of $\pi_{1} V_{11}$. Indeed, with this null initial datum, the equation (1.14) implies that $\left(\pi_{0} V_{10}\right)^{*}$ is identically zero. Then the source term in (1.13) is null, hence no secular growth for $\pi_{1} V_{11}$. The initial error is of order $O(\varepsilon)$ :

$$
\left|\left(v-v^{a}\right)(0)\right|_{L^{\infty}}=O(\varepsilon),
$$

where the functions $v$ and $v^{a}$ are respectively the exact solution and the approximate solution. We then prove the error estimate in time $O\left(\varepsilon^{\alpha}|\ln \varepsilon| / \varepsilon\right)$, for any $0<\alpha<1$ :

$$
\left|\left(v-v^{a}\right)(t)\right|=O\left(\varepsilon^{\alpha}|\ln \varepsilon|\right) .
$$

Second, for the above WKB solution, we give a stability result in diffractive times $O(1 / \varepsilon)$, corresponding to statement iii in Theorem 1.4 below. We assume additionally that the correctors are prepared as follows

$$
\left|\Pi_{s}\left(v-v^{a}\right)(0)\right|_{L^{\infty}}=O\left(\varepsilon^{2}\right),
$$

where the eigen-projectors $\Pi_{0}$ and $\Pi_{s}$ are defined later in (1.29). In the context of our WKB analysis in Section 2, the condition (1.16) is equivalent to the following condition (see also (2.51)):

$$
a_{1}(y, \theta)=\left(1-\pi_{0}\right) V_{10}(0)+\left\langle\pi_{0} V_{10}\right\rangle(0)+\tilde{a}(y, \theta),
$$

where $a_{1}$ is the first order corrector of the initial datum (1.9), and $\tilde{a}$ is a vector function that satisfies $\Pi_{s} \tilde{a}=0$. Under the preparation condition (1.16), using the specific structure of MaxwellLandau-Lifshitz (see the upcoming Section 1.1.2), we then prove that, up to times $O(1 / \varepsilon)$, the approximate solution is stable in the following sense

$$
\sup _{t \in[0, T / \varepsilon], y \in \mathbb{R}}\left|\Pi_{0}\left(v-v^{a}\right)\right|=O(\varepsilon), \quad \sup _{t \in[0, T / \varepsilon], y \in \mathbb{R}}\left|\Pi_{s}\left(v-v^{a}\right)\right|=O\left(\varepsilon^{2}\right),
$$


meaning that the initial errors (1.15) and (1.16) are essentially preserved by the dynamics of the Maxwell-Landau-Lifshitz system in the diffractive regime.

The condition (1.16) is weaker than the corresponding condition (24) in [2]. Indeed, we only give constraints on the component of the first corrector that belongs to the image of $\Pi_{s}$. Unlike the condition (24) in [2], $V_{11}(0)$ does not appear in (1.16), because we have the equality $\Pi_{s} V_{11}=0$ $($ see $(2.18))$.

\subsubsection{Maxwell-Bloch structure}

In [1, Colin (following Joly, Métivier and Rauch 4]) studies systems of the form

$$
\left\{\begin{array}{l}
\left(\partial_{t}+B_{1}\left(\partial_{x}\right)+\frac{E}{\varepsilon}\right) u=f(u, m), \\
\left(\partial_{t}+B_{2}\left(\partial_{x}\right)+\frac{F}{\varepsilon}\right) m=g(u, u) .
\end{array}\right.
$$

This operator has a block-diagonal structure which is very particular. This is the one which is used in [4. Its characteristic variety is the union of the characteristic varieties of $\partial_{t}+B_{1}\left(\partial_{x}\right)+E$ and $\partial_{t}+B_{2}\left(\partial_{x}\right)+F$. Assume the spectral decomposition

$$
B_{1}(\xi)+E / i=\sum_{j} \lambda_{j}(\xi) \Pi_{j}^{1}(\xi), B_{2}(\xi)+F / i=\sum_{j} \mu_{j}(\xi) \Pi_{j}^{2}(\xi),
$$

where $\lambda_{j}$ and $\mu_{j}$ are eigenvalues, $\Pi_{j}^{1}$ and $\Pi_{j}^{2}$ are eigenprojectors.

Colin makes a strong transparency hypothesis as in Joly, Métivier and Rauch [4] (see Assumption 2.10 in [4]), saying that

Assumption 1.1. There exists a constant $C>0$ such that for all $\xi_{1}, \xi_{2}, \eta \in \mathbb{R}^{d}$ which satisfy $\eta=\xi_{1}+\xi_{2}$, for any eigenvalues $\mu_{j}(\eta), \lambda_{j^{\prime}}\left(\xi_{1}\right), \lambda_{j^{\prime \prime}}\left(\xi_{2}\right)$ respectively of $B_{2}(\eta)+F / i, B_{1}\left(\xi_{1}\right)+E / i$ and $B_{1}\left(\xi_{2}\right)+E / i$ and for all $u, v \in \mathbb{C}^{n}$, one has

$$
\left|\Pi_{j}^{2}(\eta) g\left(\Pi_{j^{\prime}}^{1}\left(\xi_{1}\right) u, \Pi_{j^{\prime \prime}}^{1}\left(\xi_{2}\right) v\right)\right| \leq C\left|\lambda_{j^{\prime}}\left(\xi_{1}\right)+\lambda_{j^{\prime \prime}}\left(\xi_{2}\right)-\mu_{j}(\eta)\right||u||v| .
$$

We say a system has Maxwell-Bloch structure, if it is of the form (1.18) and satisfies Assumption 1.1 .

For systems with Maxwell-Bloch structure, Colin shows that a WKB expansion can be performed on (1.18), where the leading terms of the three-scale WKB solution satisfy an elliptic DaveyStewartson system which is locally well-posed, implying that an approximate solution $\left(u^{a}, m^{a}\right)$ can be constructed over times $O(1 / \varepsilon)$ (see Proposition 4.1 in [1]). Assuming additionally that the initial correctors are identically zero, that is, $(u, m)(0)=\left(u^{a}, m^{a}\right)(0)$ (which is strictly stronger than (1.16)), Colin showed a convergence result over times $O(1 / \varepsilon)$. The Maxwell-Bloch structure gives a control on the interactions of resonant waves (see Section 4.2 in [1] and Section 3.2 in this paper). In this respect, it is crucial in the derivation of error estimates in time $O(1 / \varepsilon)$.

Here, we check that the Maxwell-Landau-Lifshitz system in one space dimension (1.7) is of MaxwellBloch type.

\subsubsection{Resonances}

In order to check Assumption 1.1 on (1.4), we need to describe the resonances. For any $\xi=$ $\left(\xi_{1}, \xi_{2}, \xi_{3}\right)$, we have the spectral decomposition 


$$
\sum_{m=1}^{3} A_{m} \xi_{m}+L_{0} / i=\sum_{j=1}^{9} \lambda_{j}(\xi) \Pi_{j}(\xi)
$$

The resonance set is defined as the domain in frequency space in which the upper bound in (3.9) vanishes, that is the set of frequencies $(\xi, \eta)$ such that $\Phi_{j_{1}, j_{2}, j_{3}}(\xi, \eta)=0$, where the phases functions are defined by

$$
\Phi_{j_{1}, j_{2}, j_{3}}(\xi, \eta)=\lambda_{j_{1}}(\xi+\eta)-\lambda_{j_{2}}(\xi)-\lambda_{j_{3}}(\eta) .
$$

For (1.4), the eigen-polynomial is

$$
\operatorname{det}\left(-\lambda+\sum_{j=1}^{3} A_{j} \xi_{j}+L_{0} / i\right)=\lambda^{3}\left[\lambda^{6}-2\left(2+|\xi|^{2}\right) \lambda^{4}+\left(|\xi|^{2}\left(6+|\xi|^{2}\right)-2 \xi_{1}^{2}\right) \lambda^{2}-|\xi|^{2}\left(2|\xi|^{2}-\xi_{1}^{2}\right)\right] .
$$

It is of degree nine with three parameters $\xi_{1}, \xi_{2}$ and $\xi_{3}$. The eigenvalues $\lambda_{j}$ do not symmetrically depend on $\xi_{1}, \xi_{2}$ and $\xi_{3}$. This property makes the resonance equation $\Phi_{j_{1}, j_{2}, j_{3}}(\xi, \eta)=0$ too complicated to solve analytically. For this technical reason, we consider the one space dimensional system (1.7), in which the dispersion relation reduces to a simpler formula (see below (3.3)).

\subsection{Description of the results}

If a $C^{1}$ profile $V(t, y, \theta)$ solves the Cauchy problem

$$
\left\{\begin{array}{l}
\partial_{t} V+\frac{1}{\varepsilon} A\left(e_{1}\right) \partial_{y} V+\frac{1}{\varepsilon}\left\{-\omega \partial_{\theta}+A\left(e_{1}\right) k \partial_{\theta}+L_{0}\right\} V=B(V, V) \\
V(0, y, \theta)=a(y) e^{i \theta}+\overline{a(y)} e^{-i \theta}+\varepsilon a_{1}(y, \theta)+\varepsilon^{2} a_{2}(y, \theta)
\end{array}\right.
$$

on the time interval $[0, T / \varepsilon]$, then the function $v(t, y)$ defined as (1.10) solves the Cauchy problem (1.7)-(1.9) on the time interval $[0, T / \varepsilon]$.

Throughout the paper, we always make the following assumptions:

We assume that the phase $(\omega, k)$ satisfies $\omega \neq 0$ and the dispersion relation $\operatorname{det} L(i(\omega, k))=0$ with $L(i(\omega, k)):=\left(-i \omega+A\left(e_{1}\right) i k+L_{0}\right)=0$. This means that we choose a time frequency that is compatible with the initial space frequency. We assume that the leading term of the initial datum satisfies the polarization condition: $a(y) \in \operatorname{ker} L(i(\omega, k))$. We also assume the regularity conditions $a(y) \in H^{s}, a_{1}(y, \theta) \in H^{1}\left(\mathbb{T}_{\theta}, H^{s-1}\left(\mathbb{R}_{y}^{1}\right)\right)$ and $a_{2}(y, \theta) \in H^{1}\left(\mathbb{T}_{\theta}, H^{s-2}\left(\mathbb{R}_{y}^{1}\right)\right)$, where $s>2+1 / 2$.

In Section 2, we construct an approximate profile for (1.20) by WKB expansion. This construction relies on the structural condition (2.9), which implies the weak transparency condition of Joly, Métivier and Rauch.

Proposition 1.2. (Existence of Approximate Profile)

Under the above assumptions, there exists $V^{a} \in L^{\infty}\left([0, T / \varepsilon]_{t}, H^{1}\left(\mathbb{T}_{\theta}, H^{s-2}\left(\mathbb{R}_{y}^{1}\right)\right)\right)$ for some positive $T>0$ independent of $\varepsilon$, such that $V^{a}$ solves the following Cauchy problem on time interval $[0, T / \varepsilon]_{t}$ :

$$
\left\{\begin{array}{l}
\partial_{t} V^{a}+A\left(e_{1}\right) \partial_{y} V^{a}+\frac{1}{\varepsilon}\left\{-\omega \partial_{\theta}+A\left(e_{1}\right) k \partial_{\theta}+L_{0}\right\} V^{a}=B\left(V^{a}, V^{a}\right)+\varepsilon^{2} R \\
V^{a}(0, y, \theta)=V(0, y, \theta)+\varepsilon b(y, \theta)+\varepsilon^{2} b_{1}(y, \theta)
\end{array}\right.
$$

for some $R(t, y, \theta) \in L^{\infty}\left([0, T / \varepsilon]_{t}, H^{1}\left(\mathbb{T}_{\theta}, H^{s-2}\left(\mathbb{R}_{y}^{1}\right)\right)\right), \quad\left(b(y, \theta), b_{1}(y, \theta)\right) \in H^{1}\left(\mathbb{T}_{\theta}, H^{s-2}\left(\mathbb{R}_{y}^{1}\right)\right)$. Precisely, the function $b$ is given in (2.47) and has the property stated in Lemma 2.1; the function $R$ is given by (2.49) and has the property stated in Lemma 2.2. Moreover, the leading term of the WKB approximate profile $V^{a}$ solves a cubic Schrödinger equation. 
We remark that it does not seem possible to construct a more precise WKB solution. Indeed, for higher order terms, there arises an equation of the form

$$
\left(-\rho+\pi_{0} A\left(e_{1}\right) \pi_{0}\right) \partial_{y}\left\langle V_{20}\right\rangle=4 \operatorname{Re} \pi_{0} B\left(V_{0,-1}, V_{21}\right)+2 \operatorname{Re} \pi_{0} B\left(V_{1,-1}, V_{11}\right)-\partial_{\tau}\left\langle V_{10}\right\rangle,
$$

where the right hand side is not of the form $\partial_{y} \mathcal{R}$, then equation (1.22) is not well posed in classical Sobolev spaces. This can be contrasted with corresponding equations for lower order terms (see $(2.28)$ ), where the source terms are indeed of the form $\partial_{y} \mathcal{R}$.

For $V^{a}$ given by Proposition 1.2, defining $v^{a}:=V^{a}\left(t, x, \frac{k y-\omega t}{\varepsilon}\right)$, then $v^{a} \in L^{\infty}\left([0, T / \varepsilon]_{t} \times \mathbb{R}_{y}^{1}\right)$ and satisfies the following Cauchy problem on time interval $[0, T / \varepsilon]$ :

$$
\left\{\begin{array}{l}
\left(\partial_{t}+A\left(e_{1}\right) \partial_{y}\right) v^{a}+\frac{1}{\varepsilon} L_{0} v^{a}=B\left(v^{a}, v^{a}\right)+\varepsilon^{2} R, \\
v^{a}(0, y)=v(0, y)+\varepsilon b\left(y, \frac{k y}{\varepsilon}\right)+\varepsilon^{2} b_{1}\left(y, \frac{k y}{\varepsilon}\right) .
\end{array}\right.
$$

We then justify the WKB expansion by giving an upper bound for $\left|v-v^{a}\right|$ that is valid over the existence time for $v^{a}$ :

Theorem 1.3. Under the assumptions stated just above Proposition 1.2 , there exists $T^{*}>0$ and $0<\varepsilon_{0}<1$, such that for all $T<T^{*}$, for some constant $C=C(s, T)>0$ depending on $s$ through $\|a\|_{H^{s}}+\left\|a_{1}\right\|_{H^{2}\left(\mathbb{T}_{\theta}, H^{s-1}\right)}+\left\|a_{2}\right\|_{H^{2}\left(\mathbb{T}_{\theta}, H^{s-2}\right)}$, we have that for all $0<\varepsilon<\varepsilon_{0}$ :

i. Over the time interval $[0, T / \varepsilon]$, the Cauchy problem (1.7)-(1.9) admits a unique solution $v$ of the form $v(t, x)=V\left(t, x, \frac{k y-\omega t}{\varepsilon}\right)$, with $V(t, x, \theta) \in L^{\infty}\left([0, T / \varepsilon]_{t}, H^{1}\left(\mathbb{T}_{\theta}, H^{s-2}\left(\mathbb{R}_{y}^{1}\right)\right)\right)$.

ii. We have the following error estimates

$$
\left\|\Pi_{0}\left(v-v^{a}\right)\right\|_{L^{\infty}\left(\left[0, \frac{T}{\varepsilon}\right] \times \mathbb{R}^{1}\right)} \leq C(\varepsilon+T), \quad\left\|\Pi_{s}\left(v-v^{a}\right)\right\|_{L^{\infty}\left(\left[0, \frac{T}{\varepsilon}\right] \times \mathbb{R}^{1}\right)} \leq C \varepsilon,
$$

iii. Assuming additionally that $a_{1}$ satisfies (1.17), we have the better error estimates

$$
\left\|\Pi_{0}\left(v-v^{a}\right)\right\|_{L^{\infty}\left(\left[0, \frac{T}{\varepsilon}\right] \times \mathbb{R}^{1}\right)} \leq C \varepsilon, \quad\left\|\Pi_{s}\left(v-v^{a}\right)\right\|_{L^{\infty}\left(\left[0, \frac{T}{\varepsilon}\right] \times \mathbb{R}^{1}\right)} \leq C \varepsilon^{2} .
$$

We remark that for times just short of the diffractive time scale, that is $T=\varepsilon^{\alpha}|\ln \varepsilon|, 0<\alpha<1$, the error estimate (1.24) gives an upper bound that is $o(1)$ in the limit $\varepsilon \rightarrow 0$ :

$$
\left\|\left(v-v^{a}\right)\right\|_{L^{\infty}\left(\left[0, \varepsilon^{-1+\alpha}|\ln \varepsilon|\right] \times \mathbb{R}^{1}\right)}=O\left(\varepsilon^{\alpha}|\ln \varepsilon|\right) .
$$

The proof of Theorem (1.3) is decomposed into two steps. First in Section 3, we show that the system (1.20) has the Maxwell-Bloch structure, then by a nonlinear change of variable introduced by Joly, Métivier and Rauch in [4, we prove that the Cauchy problem (1.20) admits a unique solution over times of order $O(1 / \varepsilon)$. Then in Section 4, we prove the error estimates (1.24) and (1.25).

We conclude this introduction by setting up some notations:

For all $(\lambda, \xi) \in \mathbb{R}^{1+1}$, we introduce the matrices $L(i(\lambda, \xi))=i \lambda+A\left(e_{1}\right) i \xi+L_{0}$ and the characteristic variety $\operatorname{Char} L(i(\lambda, \xi))=\{(\lambda, \xi) \mid \operatorname{det} L(i(\lambda, \xi))=0\}$.

For $\beta=(\omega, k)$, denote $L\left(\beta \partial_{\theta}\right)=-\omega \partial_{\theta}+A\left(e_{1}\right) k \partial_{\theta}+L_{0}$ and $L_{p}=L(i p \beta)=-i p \omega+i p k A\left(e_{1}\right)+L_{0}$. Denote by $\pi_{p}$ the orthogonal projector onto ker $L_{p}$ and $L_{p}^{-1}$ the inverse of $L_{p}$, or, if $L_{p}$ is singular, its partial inverse which is defined as

$$
\pi_{p} L_{p}^{-1}=L_{p}^{-1} \pi_{p}=0, L_{p} L_{p}^{-1}=L_{p}^{-1} L_{p}=\mathrm{Id}-\pi_{p}
$$


For all $\xi \in \mathbb{R}^{1}$, we have the spectral decomposition

$$
A\left(\xi e_{1}\right)+L_{0} / i=\sum_{j=1}^{9} \lambda_{j}(\xi) \Pi_{j}(\xi) .
$$

We denote the total eigenprojectors

$$
\Pi_{0}(\xi)=\sum_{j=1}^{6} \Pi_{j}(\xi), \Pi_{s}(\xi)=\sum_{j^{\prime}=7}^{9} \Pi_{j^{\prime}}(\xi) .
$$

By direct calculation, for all $\xi \in \mathbb{R}$, we have $\lambda_{7}(\xi)=\lambda_{8}(\xi)=\lambda_{9}(\xi)=0$ and

$$
\Pi_{0}(\xi)=\operatorname{diag}\{0,1,1,0,1,1,0,1,1\}, \Pi_{s}(\xi)=\operatorname{diag}\{1,0,0,1,0,0,1,0,0\} .
$$

The projector $\Pi_{0}(\xi)$ and $\Pi_{s}(\xi)$ are actually constant matrices, independent of the variable $\xi$.

In the following, most functions and operators depend on $\varepsilon$. In this context, we typically use bounded to mean bounded uniformly in $\varepsilon \in(0,1)$. Later on, we restrict the range of $\varepsilon$ to $0<\varepsilon<\varepsilon_{0}$, for some $\varepsilon_{0}$ small enough.

\section{WKB expansion and approximate profile}

The aim of this section is to construct an approximate profile of (1.20) through WKB expansion. In the nonlinear regime of our interest, the limit equations are cubic Schrödinger equations.

\subsection{The WKB Expansion}

We look for an approximate profile $V^{a}$ of the form

$$
V^{a}(t, y, \theta)=\sum_{j=0}^{2} \varepsilon^{j} V_{j}(\varepsilon t, t, y, \theta), \quad V_{j}(\tau, t, y, \theta)=\sum_{p \in \mathbb{Z}} \varepsilon^{i p \theta} V_{j p}(\tau, t, y) .
$$

We plug (2.1) into (1.20) and cancel all the terms of orders $O\left(\varepsilon^{j}\right), j=-2,-1,0$.

Equations for the terms in $O\left(\varepsilon^{-2}\right): L\left(\beta \partial_{\theta}\right) V_{0}=\left(-\omega \partial_{\theta}+A\left(e_{1}\right) k \partial_{\theta}+L_{0}\right) V_{0}=0$. By (2.1), it amounts to solving the following equations for all $p \in \mathbb{Z}$ :

$$
L_{p} V_{0 p}=\left(-i p \omega+A\left(e_{1}\right) i p k+L_{0}\right) V_{0 p}=0 .
$$

We study (2.2) for each $p \in \mathbb{Z}$.

$\mathbf{p}=\mathbf{0}$ If $p=0$, the equation (2.2) becomes $L_{0} V_{00}=0$. Since there is no mean mode in the leading term of the initial datum (1.20), we choose naturally $V_{00}=0$. Actually, assuming the leading mean mode $V_{00}$ to be null is crucial to obtain a Davey-Stewartson approximation, as in [1] and [2] (here the Davey-Stewartson approximation degenerates into a nonlinear Schrödinger approximation).

$\mathbf{p}=1$ If $p=1$, the equation (2.2) becomes $L_{1} V_{01}=0$. By direct calculation, the dispersion relation det $L_{1}=0$ is

$$
\omega^{2}\left(1-\frac{k^{2}}{\omega^{2}}\right)^{2}=\left(2-\frac{k^{2}}{\omega^{2}}\right)^{2}
$$


We choose $\omega \neq 0$ and $k \neq 0$ that satisfy the dispersion relation (2.3). We denote

$$
\delta=-\frac{\omega\left(1-\frac{k^{2}}{\omega^{2}}\right)}{2-\frac{k^{2}}{\omega^{2}}}, \quad \gamma=1-\frac{k^{2}}{\omega^{2}},
$$

then the dispersion relation (2.3) is equivalent to

$$
k^{2}=\frac{\omega+2 \delta}{\omega+\delta} \cdot \omega^{2}, \quad \delta= \pm 1 .
$$

Through direct calculation, we find that $\operatorname{ker} L_{1}$ is a one-dimensional vector space with generator $W_{0}$ :

$$
W_{0}=\left(\begin{array}{c}
-\frac{i \delta k}{\omega} \Omega_{0} \\
\Omega_{0} \\
-\gamma \Omega_{0}
\end{array}\right), \quad \Omega_{0}=\left(\begin{array}{c}
0 \\
i \delta \\
1
\end{array}\right) .
$$

Then the orthogonal projector $\pi_{1}$ onto ker $L_{1}$ has the expression:

$$
\pi_{1} V=\frac{\left(V \mid W_{0}\right)}{\left|W_{0}\right|^{2}} W_{0}, \text { for all } V \in \mathbb{R}^{9} .
$$

where $(\cdot \mid \cdot)$ denotes the inner product in the complex vector space $\mathbb{C}^{9}$. Then the solution $V_{01}$ has the following form: for some scalar function $g$ (which is determined later by (2.40) and (2.41)):

$$
V_{01}=W_{0} g
$$

$|\mathbf{p}| \geq 2$ The equation (2.2) becomes $L_{p} V_{0 p}=0$. For nonzero $(\omega, k)$, $\operatorname{det} L_{p}=0 \Longleftrightarrow p^{2} \omega^{2}(1-$ $\left.\frac{k^{2}}{\omega^{2}}\right)^{2}=\left(2-\frac{k^{2}}{\omega^{2}}\right)^{2}$. By our choice of $(\omega, k)$ that satisfies (2.3), the matrix $L_{p}$ is invertible if $|p| \geq 2$. Then the solutions are trivial $V_{0 p}=0$, for all $|p| \geq 2$.

$p \leq-1$ By reality of the datum, the only natural choice is $V_{j, p}=\bar{V}_{j,-p}$.

Equations for the terms in $O\left(\varepsilon^{-1}\right)$. For all $p$ :

$$
\left(\partial_{t}+A\left(e_{1}\right) \partial_{y}\right) V_{0 p}+L_{p} V_{1 p}=\sum_{p_{1}+p_{2}=p} B\left(V_{0 p_{1}}, V_{0 p_{2}}\right) .
$$

$\mathbf{p}=\mathbf{0}$ If $p=0$, the equation (2.8) reduce to

$$
L_{0} V_{10}=2 B\left(V_{01}, V_{0,-1}\right)
$$

We calculate

$$
B\left(V_{01}, V_{0,-1}\right)=g \bar{g} B\left(W_{0}, \bar{W}_{0}\right)=\frac{g \bar{g}}{2}\left(\begin{array}{c}
-\gamma \Omega_{0} \times \bar{\Omega}_{0}^{0}-\gamma \bar{\Omega}_{0} \times \Omega_{0} \\
\gamma \Omega_{0} \times \bar{\Omega}_{0}+\gamma \bar{\Omega}_{0} \times \Omega_{0}
\end{array}\right)=0 .
$$

This means we have $B\left(\pi_{1} \cdot, \pi_{-1} \cdot\right)=0$. The relationship $\pi_{0} B\left(\pi_{1} \cdot, \pi_{-1} \cdot\right)=0$, which is necessary to construct the Davey-Stewartson approximate solution in Colin and Lannes [2], is the weak transparency condition introduced by Joly, Métivier and Rauch 4. Here, we even have the following stronger result

$$
B\left(\pi_{ \pm 1} \cdot, \pi_{ \pm 1} \cdot\right)=0 .
$$


Then the equation (2.9) becomes $L_{0} V_{10}=0$. We denote $\pi_{0}$ the projection onto ker $L_{0}$. Then we have $V_{10}=\pi_{0} V_{10}$. Now we calculate the vector space $\operatorname{ker} L_{0}$ and the projector $\pi_{0}$. We let $V_{10}=\left(E_{10}, H_{10}, M_{10}\right)^{t}$, where $E_{10}, H_{10}$ and $M_{10}$ are vectors in $\mathbb{R}^{3}$. The equations (1.5) and (2.9) give

$$
L_{0} V_{10}=0 \Longleftrightarrow M_{0} \times\left(H_{10}-M_{10}\right)=0 \Longleftrightarrow\left(H_{10}-M_{10}\right) / /(1,0,0)^{t} .
$$

Then we obtain a basis of $\operatorname{ker} L_{0}$ :

$$
\operatorname{ker} L_{0}=\operatorname{span}\left\{\left(\begin{array}{c}
e_{1} \\
0 \\
0
\end{array}\right),\left(\begin{array}{c}
e_{2} \\
0 \\
0
\end{array}\right),\left(\begin{array}{c}
e_{3} \\
0 \\
0
\end{array}\right),\left(\begin{array}{c}
0 \\
e_{1} \\
0
\end{array}\right),\left(\begin{array}{c}
0 \\
e_{2} \\
e_{2}
\end{array}\right),\left(\begin{array}{c}
0 \\
e_{3} \\
e_{3}
\end{array}\right),\left(\begin{array}{c}
0 \\
0 \\
e_{1}
\end{array}\right)\right\},
$$

where $e_{1}=(1,0,0)^{t}, e_{2}=(0,1,0)^{t}, e_{3}=(0,0,1)^{t}$. The orthogonal projector $\pi_{0}$ onto ker $L_{0}$ is

$$
\pi_{0}=\left(\begin{array}{ccc}
I & 0 & 0 \\
0 & J_{1} & J_{2} \\
0 & J_{2} & J_{1}
\end{array}\right)
$$

with the blocks

$$
I=\left(\begin{array}{ccc}
1 & 0 & 0 \\
0 & 1 & 0 \\
0 & 0 & 1
\end{array}\right), \quad J_{1}=\left(\begin{array}{ccc}
1 & 0 & 0 \\
0 & \frac{1}{2} & 0 \\
0 & 0 & \frac{1}{2}
\end{array}\right), \quad J_{2}=\left(\begin{array}{ccc}
0 & 0 & 0 \\
0 & \frac{1}{2} & 0 \\
0 & 0 & \frac{1}{2}
\end{array}\right)
$$

$\mathbf{p = 1}$ If $p=1$, the equation becomes $\left(\partial_{t}+A\left(e_{1}\right) \partial_{y}\right) V_{01}+L_{1} V_{11}=\sum_{p_{1}+p_{2}=1} B\left(V_{0 p_{1}}, V_{0 p_{2}}\right)$.

From the analysis for the terms in $O\left(\varepsilon^{-2}\right)$, we have that $V_{00}=0$ and $V_{0 p}=0$, for all $|p| \geq 2$, which give us $\sum_{p_{1}+p_{2}=1} B\left(V_{0 p_{1}}, V_{0 p_{2}}\right)=0$. Then the equation for $p=1$ is

$$
\left(\partial_{t}+A\left(e_{1}\right) \partial_{y}\right) V_{01}+L_{1} V_{11}=0
$$

Applying $\pi_{1}$ to (2.13), by identity $\pi_{1} L_{1}=0$, we obtain

$$
\pi_{1}\left(\partial_{t}+A\left(e_{1}\right) \partial_{y}\right) V_{01}=0,
$$

a transport equation for the leading profile. In the geometric optics approximation, the transport occurs at group velocity (See Proposition 2.6 of [8] and Proposition 2.2 in [3] ). Here we compute the transport operator $\pi_{1} A\left(e_{1}\right) \partial_{y} \pi_{1}$ explicitly. By (2.6), the equation (2.14) is equivalent to

$$
\left(\left(\partial_{t}+A\left(\partial_{x}\right)\right) V_{01} \mid W_{0}\right)=0 .
$$

By (2.5) and (2.7), we have that

$$
\left(\partial_{t} V_{01} \mid W_{0}\right)=\partial_{t} g\left|W_{0}\right|^{2}=\partial_{t} g 2\left(1+\frac{k^{2}}{\omega^{2}}+\left(1-\frac{k^{2}}{\omega^{2}}\right)^{2}\right)
$$

and that

$$
\left(A\left(e_{1}\right) \partial_{y} V_{01} \mid W_{0}\right)=\frac{4 k}{\omega} \partial_{y} g .
$$

Then the equation (2.14) becomes the following transport equation in the scalar function $g$ :

$$
\partial_{t} g+\rho \partial_{y} g=0
$$


with the group velocity

$$
\rho=\left(1+\frac{k^{2}}{\omega^{2}}+\left(1-\frac{k^{2}}{\omega^{2}}\right)^{2}\right)^{-1} \frac{2 k}{\omega} .
$$

On the other hand, by applying $L_{1}^{-1}$ to (2.13), one has

$$
\left(1-\pi_{1}\right) V_{11}=-L_{1}^{-1}\left(\left(\partial_{t}+A\left(e_{1}\right) \partial_{y}\right) V_{01}\right)=-L_{1}^{-1}\left(A\left(e_{1}\right) \partial_{y} V_{01}\right) .
$$

Explicitly, the solution of equation (2.13) is $V_{11}=\left(E_{11}, H_{11}, M_{11}\right)^{t}$ with

$$
\left\{\begin{array}{l}
E_{11}=-\frac{i \delta k}{\omega} f \Omega_{0}+\frac{\delta}{\omega}\left(-1+\frac{k \rho}{\omega}\right) \partial_{y} g \Omega_{0}, \\
H_{11}=f \Omega_{0} \\
M_{11}=-\gamma f \Omega_{0}+\frac{2 i k}{\omega^{2}}\left(-1+\frac{k \rho}{\omega}\right) \partial_{y} g \Omega_{0},
\end{array}\right.
$$

where $f$ is an unknown function that is yet to be determined (see (2.43) $)$, and is associated with the component $\pi_{1} V_{11}$ of $V_{11}$.

$\mathbf{p = 2}$ If $p=2$, the equation becomes $\left(\partial_{t}+A\left(e_{1}\right) \partial_{y}\right) V_{02}+L_{2} V_{12}=\sum_{p_{1}+p_{2}=2} B\left(V_{0 p_{1}}, V_{0 p_{2}}\right)$.

Since $V_{00}=0, V_{0 p}=0$, for all $|p| \geq 2$ and (2.9), the equation for $p=2$ is actually $L_{2} V_{12}=$ $B\left(V_{01}, V_{01}\right)=0$, which gives trivial solution $V_{12}=0$ because the matrix $L_{2}$ is invertible.

$\mathbf{p} \geq \mathbf{3}$ The equations are $\left(\partial_{t}+A\left(e_{1}\right) \partial_{y}\right) V_{0 p}+L_{p} V_{1 p}=\sum_{p_{1}+p_{2}=p} B\left(V_{0 p_{1}}, V_{0 p_{2}}\right)$, and we directly obtain the solutions $V_{1 p}=0$.

$\mathbf{p}<\mathbf{0}$ For negative $p$, we simply take $V_{j p}=\bar{V}_{j,-p}$.

Equations for the terms in $O\left(\varepsilon^{0}\right)$. For all $p$,

$$
\partial_{\tau} V_{0 p}+\left(\partial_{t}+A\left(e_{1}\right) \partial_{y}\right) V_{1 p}+L_{p} V_{2 p}=2 \sum_{p_{1}+p_{2}=p} B\left(V_{0 p_{1}}, V_{1 p_{2}}\right) .
$$

$\mathbf{p}=\mathbf{0}$ For $\mathrm{p}=0$, the equation (2.19) becomes

$$
\partial_{\tau} V_{00}+\left(\partial_{t}+A\left(e_{1}\right) \partial_{y}\right) V_{10}+L_{0} V_{20}=2 \sum_{p_{1}+p_{2}=0} B\left(V_{0 p_{1}}, V_{1 p_{2}}\right) .
$$

We calculate the right hand side

$$
\sum_{p_{1}+p_{2}=0} B\left(V_{0 p_{1}}, V_{1 p_{2}}\right)=B\left(V_{01}, V_{1,-1}\right)+B\left(V_{0,-1}, V_{11}\right)=2 \operatorname{Re} B\left(V_{0,-1}, V_{11}\right) .
$$

Together with the choice $V_{00}=0$, the equation (2.20) becomes

$$
\left(\partial_{t}+A\left(e_{1}\right) \partial_{y}\right) V_{10}+L_{0} V_{20}=4 \operatorname{Re} B\left(V_{0,-1}, V_{11}\right) .
$$

Applying $\pi_{0}$ to (2.21), we obtain that

$$
\left(\partial_{t}+\pi_{0} A\left(e_{1}\right) \partial_{y} \pi_{0}\right) \pi_{0} V_{10}=4 \pi_{0} \operatorname{Re} B\left(V_{0,-1}, V_{11}\right) .
$$


By (1.6), (2.5), (2.7), (2.11) and (2.18), we calculate the source term of (2.22):

$$
4 \pi_{0} \operatorname{Re} B\left(V_{0,-1}, V_{11}\right)=\left(\begin{array}{c}
0 \\
A_{0} \\
-A_{0}
\end{array}\right)
$$

with

$$
A_{0}=\left(\begin{array}{c}
k_{0} \\
0 \\
0
\end{array}\right), k_{0}=\frac{4 k \delta}{\omega^{2}}\left(1-\frac{k \rho}{\omega}\right) \partial_{y}|g|^{2} .
$$

By (1.5), (2.11) and (2.12), we have that

$$
\pi_{0} A\left(e_{1}\right) \pi_{0}=\left(\begin{array}{ccc}
0 & \left(-e_{1} \times\right) J_{1} & \left(-e_{1} \times\right) J_{2} \\
J_{1}\left(e_{1} \times\right) & 0 & 0 \\
J_{2}\left(e_{1} \times\right) & 0 & 0
\end{array}\right) .
$$

We denote $V_{10}=\left(E_{10}, H_{10}, M_{10}\right)$, by (2.23) and (2.25), the equation (2.22) becomes

$$
\left\{\begin{array}{l}
\partial_{t} E_{10}-\left(e_{1} \times\right) J_{1} \partial_{y} H_{10}-\left(e_{1} \times\right) J_{2} \partial_{y} M_{10}=0 \\
\partial_{t} H_{10}+J_{1}\left(e_{1} \times\right) \partial_{y} E_{10}=A_{0} \\
\partial_{t} M_{10}+J_{2}\left(e_{1} \times\right) \partial_{y} E_{10}=-A_{0} .
\end{array}\right.
$$

We now introduce a decomposition inspired from the averaging method of Lannes [5]: for any vector function $V(t, y)$, we use the notation $\langle V\rangle$ and $V^{*}$ to denote the two parts of the decomposition $V=\langle V\rangle+V^{*}$, where the part $\langle V\rangle$ satisfies the transport equation $\left(\partial_{t}+\rho \partial_{y}\right)\langle V\rangle=0$ with the same group velocity $\rho$ as the leading term $V_{01}$. To solve (2.26), it is sufficient to solve the following two systems:

$$
\left\{\begin{array}{l}
-\rho \partial_{y}\left\langle E_{10}\right\rangle-\left(e_{1} \times\right) J_{1} \partial_{y}\left\langle H_{10}\right\rangle-\left(e_{1} \times\right) J_{2} \partial_{y}\left\langle M_{10}\right\rangle=0 \\
-\rho \partial_{y}\left\langle H_{10}\right\rangle+J_{1}\left(e_{1} \times\right) \partial_{y}\left\langle E_{10}\right\rangle=A_{0} \\
-\rho \partial_{y}\left\langle M_{10}\right\rangle+J_{2}\left(e_{1} \times\right) \partial_{y}\left\langle E_{10}\right\rangle=-A_{0}
\end{array}\right.
$$

and

$$
\left\{\begin{array}{l}
\partial_{t} E_{10}^{*}-\left(e_{1} \times\right) J_{1} \partial_{y} H_{10}^{*}-\left(e_{1} \times\right) J_{2} \partial_{y} M_{10}^{*}=0, \\
\partial_{t} H_{10}^{*}+J_{1}\left(e_{1} \times\right) \partial_{y} E_{10}^{*}=0, \\
\partial_{t} M_{10}^{*}+J_{2}\left(e_{1} \times\right) \partial_{y} E_{10}^{*}=0 .
\end{array}\right.
$$

By taking $-\rho \partial_{y}$ to the second and third equations of (2.27), we obtain that

$$
\left\{\begin{array}{l}
\rho^{2} \partial_{y}^{2}\left\langle H_{10}\right\rangle+J_{1}\left(e_{1} \times\right)\left[\left(e_{1} \times\right) J_{1} \partial_{y}^{2}\left\langle H_{10}\right\rangle+\left(e_{1} \times\right) J_{2} \partial_{y}^{2}\left\langle M_{10}\right\rangle\right]=-\rho \partial_{y} A_{0}, \\
\rho^{2} \partial_{y}^{2}\left\langle M_{10}\right\rangle+J_{2}\left(e_{1} \times\right)\left[\left(e_{1} \times\right) J_{1} \partial_{y}^{2}\left\langle H_{10}\right\rangle+\left(e_{1} \times\right) J_{2} \partial_{y}^{2}\left\langle M_{10}\right\rangle\right]=\rho \partial_{y} A_{0} .
\end{array}\right.
$$

Since $V_{10}=\left(E_{10}, H_{10}, M_{10}\right) \in \operatorname{ker} L_{0}$, by (2.10), we may suppose that

$$
H_{10}=\left(\begin{array}{l}
h_{1} \\
h_{2} \\
h_{3}
\end{array}\right), M_{10}=\left(\begin{array}{c}
m_{1} \\
h_{2} \\
h_{3}
\end{array}\right) .
$$

Then by (1.8) and (2.12), the equation (2.29) becomes

$$
\left\{\begin{array}{l}
\rho^{2} \partial_{y}^{2}\left\langle h_{1}\right\rangle=-\frac{4 k \delta \rho}{\omega}\left(1-\frac{k \rho}{\omega}\right) \partial_{y}^{2}|g|^{2} \\
\left(\rho^{2}-\frac{1}{2}\right) \partial_{y}^{2}\left\langle h_{2}\right\rangle=\left(\rho^{2}-\frac{1}{2}\right) \partial_{y}^{2}\left\langle h_{3}\right\rangle=0 \\
\rho^{2} \partial_{y}^{2}\left\langle m_{1}\right\rangle=\frac{4 k \delta \rho}{\omega}\left(1-\frac{k \rho}{\omega}\right) \partial_{y}^{2}|g|^{2}
\end{array}\right.
$$


A solution to (2.31) is

$$
\left\langle h_{1}\right\rangle=-\frac{4 k \delta \rho}{\omega}\left(1-\frac{k \rho}{\omega}\right)|g|^{2}, \quad\left\langle m_{1}\right\rangle=\frac{4 k \delta}{\omega \rho}\left(1-\frac{k \rho}{\omega}\right)|g|^{2}, \quad\left\langle h_{2}\right\rangle=\left\langle h_{3}\right\rangle=0 .
$$

Plugging (2.30) and (2.32) into the first equation of (2.27), we have that $\partial_{y}\left\langle E_{10}\right\rangle=0$ which gives the trivial solution $\left\langle E_{10}\right\rangle=0$.

We solve the equation (2.28) by choosing the trivial solution $E_{10}^{*}=H_{10}^{*}=M_{10}^{*}=0$. By doing so, we will not see a source term in (2.38) and there will be no secular growth for $\pi_{1} V_{11}$.

With our choice for $V_{10}^{*}$ and $\left\langle V_{10}\right\rangle$, we have a solution to (2.22):

$$
V_{10}=\left(\begin{array}{c}
E_{10} \\
H_{10} \\
M_{10}
\end{array}\right)=\frac{4 k \delta}{\omega \rho}\left(1-\frac{k \rho}{\omega}\right)\left(\begin{array}{c}
0 \\
-e_{1} \\
e_{1}
\end{array}\right)|g|^{2},
$$

where the vector $e_{1}$ is given in (1.8).

Back to (2.21), by (2.7), (2.18) and (2.33), we obtain that $L_{0} V_{20}=0$, which admits the trivial solution $V_{20}=0$. Now equation (2.21) is solved.

$\mathbf{p}=\mathbf{1}$ For $\mathrm{p}=1$, the equation (2.19) becomes

$$
\partial_{\tau} V_{01}+\left(\partial_{t}+A\left(e_{1}\right)\right) \partial_{y} V_{11}+L_{1} V_{21}=2 B\left(V_{01}, V_{10}\right) .
$$

Applying $\pi_{1}$ to (2.34) gives

$$
\partial_{\tau} V_{01}+\pi_{1}\left(\partial_{t}+A\left(e_{1}\right)\right) \partial_{y} V_{11}=2 \pi_{1} B\left(V_{01}, V_{10}\right) .
$$

By (2.17), we have that

$$
\pi_{1}\left(\partial_{t}+A\left(e_{1}\right)\right) \partial_{y} V_{11}=\left(\partial_{t}+\pi_{1} A\left(e_{1}\right) \partial_{y} \pi_{1}\right) \pi_{1} V_{11}-\pi_{1} A\left(e_{1}\right) \partial_{y} L_{1}^{-1}\left(A\left(e_{1}\right) \partial_{y} V_{01}\right),
$$

We already have $\pi_{1} A\left(e_{1}\right) \partial_{y} \pi_{1}=\rho \partial_{y}$ with group velocity $\rho$ in (2.16). In diffractive optics approximation, we have $-\pi_{1} A\left(e_{1}\right) \partial_{y} L_{1}^{-1} A\left(e_{1}\right) \partial_{y}=-\frac{i}{2} \omega^{\prime \prime}(k) \partial_{y}^{2}$, which gives a Schrödinger equation. (See Proposition 2.6 in [8] or Proposition 4.1 in [3]). Here we compute the Schrödinger operator $-\pi_{1} A\left(e_{1}\right) \partial_{y} L_{1}^{-1} A\left(e_{1}\right) \partial_{y}$ explicitly.

By (2.6), the equation (2.35) is equivalent to

$$
\left(\partial_{\tau} V_{01} \mid W_{0}\right)+\left(\left(\partial_{t}+A\left(e_{1}\right) \partial_{y}\right) V_{11} \mid W_{0}\right)=2\left(B\left(V_{01}, V_{10}\right) \mid W_{0}\right) .
$$

Since $V_{01}=g W_{0}$, we have $\left(\partial_{\tau} V_{01} \mid W_{0}\right)=\partial_{\tau} g\left|W_{0}\right|^{2}$. By (2.5), (2.15) and (2.18), we have

$$
\left(\partial_{t} V_{11} \mid W_{0}\right)=\left|W_{0}\right|^{2} \partial_{t} f+\frac{2 i k \rho}{\omega^{2}}\left(1-\frac{k \rho}{\omega}\right)(1-2 \gamma) \partial_{y}^{2} g
$$

and

$$
\left(A\left(e_{1}\right) \partial_{y} V_{11} \mid W_{0}\right)=\frac{4 k}{\omega} \partial_{y} f-\frac{2 i}{\omega}\left(1-\frac{k \rho}{\omega}\right) \partial_{y}^{2} g .
$$

For the right hand side of (2.36), by (1.6), (2.6) and (2.33),

$$
2\left(B\left(V_{01}, V_{10}\right) \mid W_{0}\right)=\frac{8 i k}{\omega \rho}\left(1-\frac{k \rho}{\omega}\right)\left(1-\gamma^{2}\right) g|g|^{2} .
$$


We denote the real constant

$$
\nu:=\frac{\left|W_{0}\right|^{2}}{2}=1+\frac{k^{2}}{\omega^{2}}+\left(1-\frac{k^{2}}{\omega^{2}}\right)^{2},
$$

then the equation (2.35) is equivalent to

$$
\partial_{\tau} g+\frac{i}{\nu \omega}\left[\frac{k \rho}{\omega}(1-2 \gamma)-1\right]\left(1-\frac{k \rho}{\omega}\right) \partial_{y}^{2} g+\left(\partial_{t}+\rho \partial_{y}\right) f=\frac{4 i k}{\omega \rho}\left(1-\frac{k \rho}{\omega}\right)\left(1-\gamma^{2}\right) g|g|^{2} .
$$

We decompose (2.37) into two equations: a transport equation in $f$ related to $\pi_{1} V_{11}$ :

$$
\left(\partial_{t}+\rho \partial_{y}\right) f=0,
$$

and a cubic Schrödinger equation in $g$ related to $V_{01}$ :

$$
\partial_{\tau} g+i \nu_{1} \partial_{y}^{2} g=i \nu_{2} g|g|^{2}
$$

where the real constants $\nu_{1}$ and $\nu_{2}$ are defined as

$$
\nu_{1}=\frac{1}{\nu \omega}\left[\frac{k \rho}{\omega}(1-2 \gamma)-1\right]\left(1-\frac{k \rho}{\omega}\right), \quad \nu_{2}=\frac{4 k}{\omega \rho}\left(1-\frac{k \rho}{\omega}\right)\left(1-\gamma^{2}\right) .
$$

Since the scalar function $g$ satisfies the transport equation (2.15), then $g$ is of the form

$$
g(\tau, t, y)=\left.g_{1}(\tau, z)\right|_{z=y-\rho t},
$$

for some scalar function $g_{1}$. By (2.39), the function $g_{1}(\tau, z)$ solves

$$
\partial_{\tau} g_{1}+i \nu_{1} \partial_{z}^{2} g_{1}=i \nu_{2} g_{1}\left|g_{1}\right|^{2} .
$$

By the classical theory of semilinear Schrödinger equations, given any regular initial datum $g_{1}(\tau, z)=$ $a_{0}(z) \in H^{s}, s>2+1 / 2$, the equation (2.41) admits a unique local-in-time solution, with existence time $T_{1}^{*}>0$ independent of $\varepsilon$, depending on the $H^{s}$ norm of $a_{0}$. One has the following estimate for all $T<T_{1}^{*}$ :

$$
\sup _{0 \leq \tau \leq T}\left\|g_{1}(\tau, \cdot)\right\|_{H^{s}} \leq C(T)<+\infty .
$$

Then there exists a unique solution $g \in L^{\infty}\left(\left[0, T_{s}^{*}\left[\tau \times \mathbb{R}_{t}, H^{s}\right)\right.\right.$ to (2.15) and (2.39) which takes the form (2.40).

Back to equation (2.34), one can obtain the solution $V_{21}=\left(E_{21}, H_{21}, M_{21}\right)$ in terms of the functions $f, g$ and an unknown scalar function $h$ :

$$
\left\{\begin{array}{l}
E_{21}=-\frac{i \delta k}{\omega} h \Omega_{0}+\frac{\delta}{\omega}\left(-1+\frac{k \rho}{\omega}\right) \partial_{y} f \Omega_{0}+i \nu_{3} \partial_{y}^{2} g \Omega_{0}, \\
H_{21}=h \Omega_{0}, \\
M_{21}=-\gamma h \Omega_{0}+\frac{2 i k}{\omega^{2}}\left(-1+\frac{k \rho}{\omega}\right) \partial_{y} f \Omega_{0}+\nu_{4} \partial_{y}^{2} g \Omega_{0} .
\end{array}\right.
$$

The constants $m_{3}$ and $m_{4}$ are real and defined as

$$
\nu_{3}=\frac{\delta \rho}{2 \omega^{2}}\left(\frac{k \rho}{\omega}-1\right)\left(\frac{k \rho}{\omega}(1-2 \lambda)-3\right), \quad \nu_{4}=-\frac{1}{\omega^{2}}\left(\frac{k \rho}{\omega}-1\right)\left(\frac{4 k \rho}{\omega}-\left(\frac{k \rho}{\omega}\right)^{2}(1-2 \lambda)-1\right) .
$$

We define $h$ and $f$ by

$$
h=0, f=i\left[\frac{2 k}{\omega^{2}}\left(-1+\frac{k \rho}{\omega}\right)\right]^{-1} \nu_{4} \partial_{y} g .
$$


Because the function $g$ satisfies the transport equation (2.15), the function $f$ defined by (2.43) is a solution to (2.38). The point is that, with this choice of $h$ and $f$, there holds Lemma 2.2 below. This is the key property that, together with Lemma 2.1. allows the change of variable (4.12) in Section 4.2. By (2.42) and (2.43), we directly have

$$
H_{21}=M_{21}=0 .
$$

$\mathbf{p = 2}$ For $p=2$, the equation (2.19) becomes $L_{2} V_{22}=2 B\left(V_{01}, V_{11}\right)$. By (2.7) and (2.18), we have $B\left(V_{01}, V_{11}\right)=0$, and by the invertibility of $L_{2}$, the unique solution is $V_{22}=0$.

$\mathbf{p} \geq \mathbf{3}$ For $p \geq 3$, to equation (2.19): $V_{2 p}=0$.

\subsection{The approximate solution and the remainder}

The vector space $\operatorname{ker} L(i(\omega, k))$ is one-dimensional with generator $W_{0}$ defined in (2.5). The initial leading amplitude $a(y) \in \operatorname{ker} L(i(\omega, k))$ and $a \in H^{s}$ with $s>2+1 / 2$, so there exists a scalar function $a_{0}(y) \in H^{s}$ such that $a(y)=a_{0}(y) W_{0}$.

Given initial datum $g(0,0, y)=a_{0}(y)$, the transport equation (2.15) and the Schrödinger equation (2.39) admit a unique solution which takes the form (2.40) over time interval $\left[0, T_{1}^{*}\right.$ ), where $T_{1}^{*}>0$ independent of $\varepsilon$ is the existence time of the cubic Schrödinger equation (2.40). For any $T<T_{1}^{*}$ $g \in L^{\infty}\left([0, T]_{\tau} \times \mathbb{R}_{t}, H^{s}\right)$. We then choose $(f, h)$ as in (2.43), one has $f \in L^{\infty}\left([0, T]_{\tau} \times \mathbb{R}_{t}, H^{s-1}\right)$ and the following estimates for any $T<T_{1}^{*}$ :

By (2.7), we have $V_{01} \in L^{\infty}\left([0, T]_{\tau} \times \mathbb{R}_{t}, H^{s}\right)$. By (2.18), we have $V_{11} \in L^{\infty}\left([0, T]_{\tau} \times \mathbb{R}_{t}, H^{s-1}\right)$. By (2.33), we have $V_{10} \in L^{\infty}\left([0, T]_{\tau} \times \mathbb{R}_{t}, H^{s}\right)$. By (2.42), we have $V_{21} \in L^{\infty}\left([0, T]_{\tau} \times \mathbb{R}_{t}, H^{s-2}\right)$.

We then let

$$
\left\{\begin{array}{l}
V^{0}(t, y, \theta):=V_{01}(\varepsilon t, t, y) e^{i \theta}+\bar{V}_{01}(\varepsilon t, t, y) e^{-i \theta} \\
V^{1}(t, y, \theta):=\left(V_{10}+V_{11} e^{i \theta}+\bar{V}_{11} e^{-i \theta}\right)(\varepsilon t, t, y) \\
V^{2}(t, y, \theta):=\left(V_{21} e^{i \theta}+\bar{V}_{21} e^{-i \theta}\right)(\varepsilon t, t, y) .
\end{array}\right.
$$

Define $V^{a}(t, y, \theta)$ as

$$
V^{a}=V^{0}+\varepsilon V^{1}+\varepsilon^{2} V^{2}
$$

then the profile $V^{a}$ satisfies

$$
\left\{\begin{array}{l}
\partial_{t} V^{a}+A\left(e_{1}\right) \partial_{y} V^{a}+\frac{1}{\varepsilon}\left\{-\omega \partial_{\theta}+A\left(e_{1}\right) k \partial_{\theta}+L_{0}\right\} V^{a}=B\left(V^{a}, V^{a}\right)+\varepsilon^{2} R \\
V^{a}(0, y, \theta)=V(0, y, \theta)+\varepsilon b(y, \theta)+\varepsilon^{2} b_{1}(y, \theta)
\end{array}\right.
$$

over long time interval $\left[0, T_{1}^{*} / \varepsilon\left[\right.\right.$. The initial perturbations $b$ and $b_{1}$ have the expressions

$$
\begin{aligned}
& b(y, \theta)=-a_{1}(y, \theta)+\left(V_{10}+V_{11} e^{i \theta}+\bar{V}_{11} e^{-i \theta}\right)(0,0, y), \\
& b_{1}(y, \theta)=-a_{2}(y, \theta)+\left(V_{21} e^{i \theta}+\bar{V}_{21} e^{-i \theta}\right)(0,0, y),
\end{aligned}
$$

and the remainder $R(t, y, \theta)$ is defined as

$$
R=-2 B\left(V^{0}, V^{2}\right)-B\left(V^{1}, V^{1}\right)-\varepsilon 2 B\left(V^{1}, V^{2}\right)-\varepsilon^{2} B\left(V^{2}, V^{2}\right) .
$$

For any $T<T_{1}^{*}$, we have the following estimates

$$
R \in L^{\infty}\left([0, T / \varepsilon]_{t}, H^{1}\left(\mathbb{T}_{\theta}, H^{s-2}\left(\mathbb{R}_{y}^{1}\right)\right)\right),\left(b, b_{1}\right) \in H^{1}\left(\mathbb{T}_{\theta}, H^{s-2}\left(\mathbb{R}_{y}^{1}\right)\right) .
$$

At this stage, Proposition 1.2 is proved.

We show more properties of $R$ and $b$ in the following two lemmas, according to the WKB expansion in Section 2.1. 
Lemma 2.1. For initial perturbations $b$ and $b_{1}$ defined in (2.47), we have the equivalence

$$
\left|\Pi_{s}\left(V(0)-V^{a}(0)\right)\right|_{H^{1}\left(\mathbb{T}_{\theta}, H^{s-2}\left(\mathbb{R}_{y}^{1}\right)\right)}=O\left(\varepsilon^{2}\right) \Longleftrightarrow \Pi_{s} b=0 .
$$

Moreover, there holds $\Pi_{s} b=0$ if and only if the initial corrector $a_{1}$ in (1.9) is given by (2.51) below.

Proof. By (1.29) and (2.18), we have that $\Pi_{s} V_{11}=0$. Then $\Pi_{s} b=0$, by (2.47), it is necessary and sufficient to have

$$
a_{1}(y, \theta)=V_{10}(0,0, y)+\tilde{a}_{1}(y, \theta)=\frac{4 k \delta}{\omega \rho}\left(1-\frac{k \rho}{\omega}\right)\left(\begin{array}{c}
0 \\
-e_{1} \\
e_{1}
\end{array}\right)\left|a_{0}(y)\right|^{2}+\tilde{a}_{1}(y, \theta),
$$

where $\tilde{a}_{1}$ satisfies $\Pi_{s} \tilde{a}_{1}=0$. The projector $\Pi_{s}$ is defined in (1.29). Direct calculation gives the equivalence (2.50)

Lemma 2.2. With $f$ and $h$ given by (2.43), the remainder $R$ defined in (2.49) satisfies

$$
\Pi_{s} R=O(\varepsilon) \text { in } L^{\infty}\left([0, T / \varepsilon]_{t}, H^{1}\left(\mathbb{T}_{\theta}, H^{s-2}\left(\mathbb{R}_{y}^{1}\right)\right)\right), \quad \text { for any } T<T_{1}^{*} .
$$

Proof. By (2.45), we have

$$
B\left(V^{0}, V^{2}\right)=B\left(V_{11}, \bar{V}_{21}\right)+e^{2 i \theta} B\left(V_{11}, V_{21}\right)+c . c .
$$

By (2.44), a consequence of (2.43), the components $H_{21}$ and $M_{21}$ of $V_{21}$ satisfy $H_{21}=M_{21}=0$. Then by the definition of $B$ in (1.6), it is easy to obtain $B\left(V^{0}, V^{2}\right)=0$. Also by (2.45), we have that

$$
B\left(V^{1}, V^{1}\right)=B\left(V_{10}, V_{10}\right)+B\left(V_{11}, \bar{V}_{11}\right)+e^{i \theta} B\left(V_{10}, V_{11}\right)+e^{i 2 \theta} B\left(V_{11}, V_{11}\right)+c . c .
$$

By (1.6), (2.7), (2.18) and (2.33), direct calculation gives

$$
B\left(V_{10}, V_{10}\right)=B\left(V_{11}, V_{11}\right)=0, \quad B\left(V_{11}, \bar{V}_{11}\right)=\left(\begin{array}{c}
0 \\
A_{1} \\
-A_{1}
\end{array}\right),
$$

where

$$
A_{1}=\frac{i k}{\omega^{2}}\left(\frac{k \rho}{\omega}-1\right)\left(\bar{f} \partial_{y} g+f \partial_{y} \bar{g}\right)\left(\Omega_{0} \times \bar{\Omega}_{0}\right) .
$$

With $f$ given in (2.43), it is easy to check that $\bar{f} \partial_{y} g+f \partial_{y} \bar{g}=0$, and then $B\left(V_{11}, \bar{V}_{11}\right)=0$. Through direct calculation, we have $\Pi_{s} B\left(V_{10}, V_{11}\right)=0$. Then $\Pi_{s} B\left(V^{1}, V^{1}\right)=0$, and by the definition of $R$ in (2.49), we have

$$
\Pi_{s} R=-\varepsilon \Pi_{s}\left(2 B\left(V^{1}, V^{2}\right)+\varepsilon B\left(V^{2}, V^{2}\right)\right) .
$$

The lemma is proved.

We constructed a WKB solution $v^{a}$ to (1.23). We now investigate the question whether the WKB solution actually approximates the exact solution over an interval of existence. 


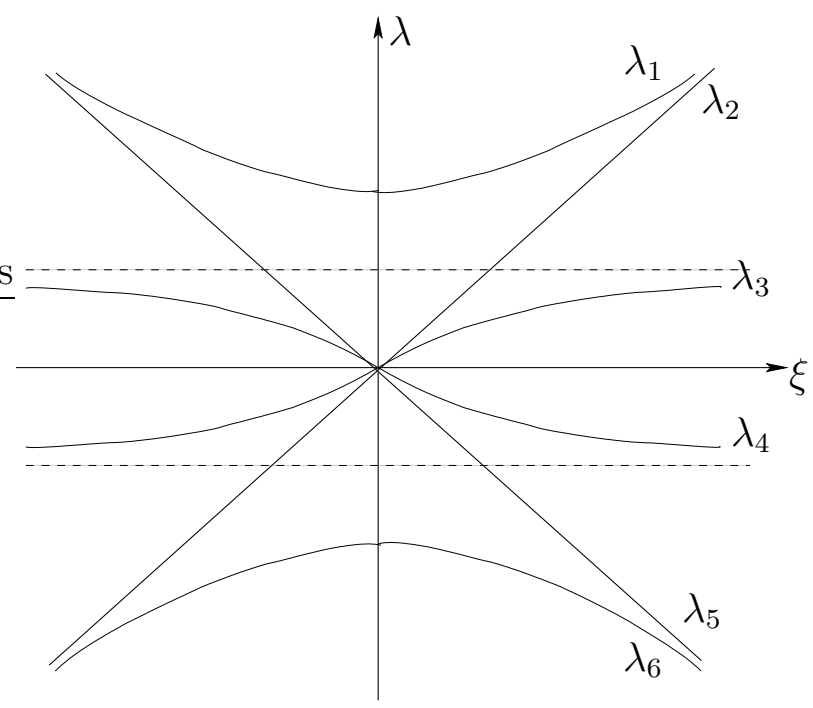

Figure 1: The characteristic variety.

\section{Maxwell-Bloch structure and long time exis- tence.}

In this section, we first show that the system (1.20) has the Maxwell-Bloch structure, then prove a long time existence result by normal form reduction.

\subsection{Spectral decomposition}

As mentioned in Section 1.2, for the symmetric matrix $A\left(e_{1}\right) \xi+L_{0} / i$, we have the following spectral decomposition

$$
A\left(e_{1}\right) \xi+\frac{L_{0}}{i}=\sum_{j=1}^{9} \lambda_{j}(\xi) \Pi_{j}(\xi) .
$$

The characteristic variety (that is, the union of the graphs $\xi \mapsto \lambda_{j}(\xi)$ ) is pictured on figure 1 . We have that for any $\xi \in \mathbb{R}, 1 \leq j \leq 6$, and $7 \leq j^{\prime} \leq 9$ :

$$
\lambda_{1}(\xi) \geq 2, \quad \lambda_{2}(\xi) \geq 0, \quad 0 \leq \lambda_{3}(\xi)<1, \quad \lambda_{j}(\xi)=-\lambda_{7-j}(\xi), \quad \lambda_{j^{\prime}}(\xi)=0 .
$$

For $1 \leq j \leq 6$, we have the dispersion relations

$$
\xi^{2}=\frac{\lambda_{j}(\xi)+2 \delta_{j}}{\lambda_{j}(\xi)+\delta_{j}} \lambda_{j}^{2}(\xi), \quad \delta_{j}=(-1)^{j}
$$

In order to reveal the Maxwell-Bloch structure, we define

$$
V_{0}=\Pi_{0} V=\sum_{j=1}^{6} \Pi_{j}\left(\varepsilon \mathcal{D}_{y}+k \mathcal{D}_{\theta}\right) V, \quad V_{s}=\Pi_{s} V=\sum_{j=7}^{9} \Pi_{j}\left(\varepsilon \mathcal{D}_{y}+k \mathcal{D}_{\theta}\right) V,
$$


where the total projectors $\Pi_{0}$ and $\Pi_{s}$ are defined in (1.28) and the notation $\mathcal{D}:=\partial / i$. By (1.29), the projector $\Pi_{0}$ and $\Pi_{s}$ are actually constant matrices. By equation (1.11) in $V$, the system in $\left(V_{0}, V_{s}\right)$ is

$$
\left\{\begin{array}{l}
\partial_{t} V_{0}+\frac{i}{\varepsilon} \mathcal{A}_{0} V_{0}-\frac{\omega \partial_{\theta}}{\varepsilon} V_{0}=\Pi_{0} B\left(V_{0}+V_{s}, V_{0}+V_{s}\right), \\
\partial_{t} V_{s}-\frac{\omega \partial_{\theta}}{\varepsilon} V_{s}=\Pi_{s} B\left(V_{0}+V_{s}, V_{0}+V_{s}\right),
\end{array}\right.
$$

where the Fourier multiplier

$$
\mathcal{A}_{0}=\sum_{j=1}^{6} \lambda_{j}\left(\varepsilon \mathcal{D}_{y}+k \mathcal{D}_{\theta}\right) \Pi_{j}\left(\varepsilon \mathcal{D}_{y}+k \mathcal{D}_{\theta}\right)
$$

By (1.6) and (1.29), we have

$$
\Pi_{0} B\left(\Pi_{0}, \Pi_{0}\right)=\Pi_{0} B\left(\Pi_{s}, \Pi_{s}\right)=\Pi_{s} B\left(\Pi_{s}, \Pi_{s}\right)=\Pi_{s} B\left(\Pi_{0}, \Pi_{s}\right)=0 .
$$

Then the equation (3.5) becomes

$$
\left\{\begin{array}{l}
\partial_{t} V_{0}+\frac{i}{\varepsilon} \mathcal{A}_{0} V_{0}-\frac{\omega \partial_{\theta}}{\varepsilon} V_{0}=2 \Pi_{0} B\left(V_{0}, V_{s}\right) \\
\partial_{t} V_{s}-\frac{\omega \partial_{\theta}}{\varepsilon} V_{s}=\Pi_{s} B\left(V_{0}, V_{0}\right)
\end{array}\right.
$$

\subsection{Strong transparency and normal form reduction}

The following proposition states that the system (3.8) satisfies a strong transparency hypothesis.

Proposition 3.1. There exists a constant $C>0$ such that for all $\xi, \eta \in \mathbb{R}$, for any eigenvalues $\lambda_{j}(\xi), \lambda_{j^{\prime}}(\eta), 1 \leq j, j^{\prime} \leq 6$, and for all $u, v \in \mathbb{C}^{9}$, one has

$$
\left|\Pi_{s} B\left(\Pi_{j}(\xi) u, \Pi_{j^{\prime}}(\eta) v\right)\right| \leq C\left|\lambda_{j}(\xi)+\lambda_{j^{\prime}}(\eta)\right||u||v| .
$$

Proof. By direct calculation, for any $1 \leq j \leq 6$, the eigenprojector $\Pi_{j}$ has the formula

$$
\Pi_{j}(\xi)=\frac{1}{\left|Q_{j}\right|^{2}}\left(\cdot \mid Q_{j}(\xi)\right) Q_{j}(\xi)
$$

where

$$
Q_{j}=\left(\begin{array}{c}
\frac{-i \delta_{j} \xi}{\lambda_{j}} \Omega_{j} \\
\Omega_{j} \\
-\gamma_{j} \Omega_{j}
\end{array}\right), \quad \Omega_{j}=\left(\begin{array}{c}
0 \\
i \delta_{j} \\
1
\end{array}\right), \quad \gamma_{j}=1-\frac{\xi^{2}}{\lambda_{j}^{2}}, \delta_{j}=(-1)^{j},
$$

and the couple $\left(\lambda_{j}, \xi\right)$ satisfies the dispersion relation (3.3). We then compute

$$
\Pi_{s} B\left(Q_{j}(\xi), Q_{j^{\prime}}(\eta)\right)=\frac{i}{2}\left(\gamma_{j}(\xi)-\gamma_{j^{\prime}}(\eta)\left(\delta_{j}-\delta_{j^{\prime}}\right)\left(\begin{array}{c}
0 \\
-e_{1} \\
e_{1}
\end{array}\right) .\right.
$$

By (3.11), we have

$$
\begin{aligned}
\gamma_{j}(\xi)-\gamma_{j^{\prime}}(\eta) & =\left(1-\frac{\xi^{2}}{\lambda_{j}(\xi)}\right)-\left(1-\frac{\eta^{2}}{\lambda_{j^{\prime}}(\eta)}\right) \\
& =\left(1-\frac{\lambda_{j}(\xi)+2 \delta_{j}}{\lambda_{j}(\xi)+\delta_{j}}\right)-\left(1-\frac{\lambda_{j^{\prime}}(\eta)+2 \delta_{j^{\prime}}}{\lambda_{j^{\prime}}(\eta)+\delta_{j^{\prime}}}\right) \\
& =\frac{-\delta_{j}\left(\lambda_{j^{\prime}}(\eta)-\delta_{j} \delta_{j^{\prime}} \lambda_{j}(\xi)\right)}{\left(\lambda_{j}(\xi)+\delta_{j}\right)\left(\lambda_{j^{\prime}}(\eta)+\delta_{j^{\prime}}\right)} .
\end{aligned}
$$


Recall that $\delta_{j}$ and $\delta_{j^{\prime}}$ can only take the value 1 or -1 . If $\delta_{j^{\prime}}$ and $\delta_{j}$ have the same sign, by (3.12), we have $\Pi_{s} B\left(Q_{j}(\xi), Q_{j^{\prime}}(\eta)\right)=0$; if $\delta_{j^{\prime}}$ and $\delta_{j}$ have the opposite sign, by (3.13), we have

$$
\Pi_{s} B\left(Q_{j}(\xi), Q_{j^{\prime}}(\eta)\right)=\frac{i\left(\lambda_{j^{\prime}}+\lambda_{j}\right)}{\left(\lambda_{j}+\delta_{j}\right)\left(\lambda_{j^{\prime}}+\delta_{j^{\prime}}\right)}\left(\begin{array}{c}
0 \\
-e_{1} \\
e_{1}
\end{array}\right) .
$$

By (3.10) and direct calculation, the equation (3.9) follows.

With (3.8) and Proposition 3.1, we see that system 3.8 has the Maxwell-Bloch structure (terminology introduced in Section 1.1.2). This allows to use a nonlinear change of variable introduced by Joly, Métivier and Rauch 4. Together with the preparation condition $\Pi_{s} V(0)=O(\varepsilon)$, this gives existence in time $O(1 / \varepsilon)$ for the solution to the Cauchy problem (3.8). First, changing variable $V_{s}=\varepsilon W_{s}$ gives

$$
\left\{\begin{array}{l}
\partial_{t} V_{0}+\frac{i}{\varepsilon} \mathcal{A}_{0} V_{0}-\frac{\omega \partial_{\theta}}{\varepsilon} V_{0}=2 \varepsilon \Pi_{0} B\left(V_{0}, W_{s}\right), \\
\partial_{t} W_{s}-\frac{\omega \partial_{\theta}}{\varepsilon} W_{s}=\frac{1}{\varepsilon} \Pi_{s} B\left(V_{0}, V_{0}\right) .
\end{array}\right.
$$

Then we introduce the nonlinear change of variable

$$
N=W_{s}-J\left(V_{0}, V_{0}\right),
$$

where the symmetric bilinear form $J$ has the following form

$$
J\left(\sum_{p \in \mathbb{Z}} u_{p} e^{i p \theta}, \sum_{q \in \mathbb{Z}} v_{q} e^{i q \theta}\right)=\sum_{p, q} J_{p q}\left(u_{p}, v_{q}\right) e^{i(p+q) \theta},
$$

for some $J_{p q}$ to be determined below. The equation in $N$ is

$$
\begin{aligned}
\partial_{t} N & -\frac{\omega \partial_{\theta}}{\varepsilon} N=\partial_{t} W_{s}-\frac{\omega \partial_{\theta}}{\varepsilon} W_{s}-J\left(\partial_{t} V_{0}-\frac{\omega \partial_{\theta}}{\varepsilon} V_{0}, V_{0}\right)-J\left(V_{0}, \partial_{t} V_{0}-\frac{\omega \partial_{\theta}}{\varepsilon} V_{0}\right) \\
= & \frac{1}{\varepsilon} \Pi_{s} B\left(V_{0}, V_{0}\right)-J\left(-\frac{i}{\varepsilon} \sum_{j=1}^{6} \lambda_{j}\left(\varepsilon \mathcal{D}_{y}+k \mathcal{D}_{\theta}\right) \Pi_{j}\left(\varepsilon \mathcal{D}_{y}+k \mathcal{D}_{\theta}\right) V_{0}, V_{0}\right) \\
& -J\left(V_{0},-\frac{i}{\varepsilon} \sum_{j=1}^{6} \lambda_{j}\left(\varepsilon \mathcal{D}_{y}+k \mathcal{D}_{\theta}\right) \Pi_{j}\left(\varepsilon \mathcal{D}_{y}+k \mathcal{D}_{\theta}\right) V_{0}\right)-2 J\left(2 \varepsilon \Pi_{0} B\left(V_{0}, W_{s}\right), V_{0}\right) .
\end{aligned}
$$

We choose the bilinear operator $J$ to eliminate the singular term of order $O(1 / \varepsilon)$ in the above equation. We consider the following equation

$$
\begin{aligned}
\Pi_{s} B\left(V_{0}, V_{0}\right)= & -i J\left(\sum_{j=1}^{6} \lambda_{j}\left(\varepsilon \mathcal{D}_{y}+k \mathcal{D}_{\theta}\right) \Pi_{j}\left(\varepsilon \mathcal{D}_{y}+k \mathcal{D}_{\theta}\right) V_{0}, V_{0}\right) \\
& -i J\left(V_{0}, \sum_{j=1}^{6} \lambda_{j}\left(\varepsilon \mathcal{D}_{y}+k \mathcal{D}_{\theta}\right) \Pi_{j}\left(\varepsilon \mathcal{D}_{y}+k \mathcal{D}_{\theta}\right) V_{0}\right) .
\end{aligned}
$$

Equivalently, in Fourier, for all $\left(\xi, \eta, p, q, j, j^{\prime}\right)$ :

$$
\begin{aligned}
\Pi_{s} B\left(\Pi_{j}(\varepsilon \eta+k p) V_{0 p}, \Pi_{j^{\prime}}(\varepsilon(\xi-\eta)+k q) V_{0 q}\right) & \\
= & -i \lambda_{j}(\varepsilon \eta+k p) J_{p q}\left(\Pi_{j}(\varepsilon \eta+k p) V_{0 p}, \Pi_{j^{\prime}}(\varepsilon(\xi-\eta)+k q) V_{0 q}\right) \\
& -i \lambda_{j}(\varepsilon(\xi-\eta)+k q) J_{p q}\left(\Pi_{j}(\varepsilon \eta+k p) V_{0 p}, \Pi_{j^{\prime}}(\varepsilon(\xi-\eta)+k q) V_{0 q}\right) .
\end{aligned}
$$


A solution to the above equation is given by

$$
J_{p q}\left(\Pi_{j}(\xi) a, \Pi_{j^{\prime}}(\eta) b\right):=i \sum_{j=1}^{6} \sum_{j^{\prime}=1}^{6} \frac{\Pi_{s} B\left(\Pi_{j}(\xi) a, \Pi_{j^{\prime}}(\eta) b\right)}{\lambda_{j}(\xi)+\lambda_{j^{\prime}}(\eta)}, \text { for all } a, b \in \mathbb{C}^{9} .
$$

By Proposition 3.1, $J$ is well defined and is bounded from $H^{s_{1}}\left(\mathbb{T}_{\theta}, H_{y}^{s_{2}}\right) \times H^{s_{1}}\left(\mathbb{T}_{\theta}, H_{y}^{s_{2}}\right)$ to $H^{s_{1}}\left(\mathbb{T}_{\theta}, H_{y}^{s_{2}}\right)$ provided $s_{1}>1 / 2, s_{2}>1 / 2$. The system in $\left(V_{0}, N\right)$ is now

$$
\left\{\begin{array}{l}
\partial_{t} V_{0}+\frac{i}{\varepsilon} \mathcal{A}_{0} V_{0}-\frac{\omega \partial_{\theta}}{\varepsilon} V_{0}=2 \varepsilon \Pi_{0} B\left(V_{0}, N+J\left(V_{0}, V_{0}\right)\right), \\
\partial_{t} N-\frac{\omega \partial_{\theta}}{\varepsilon} N=-2 \varepsilon J\left(\Pi_{0} B\left(V_{0}, N+J\left(V_{0}, V_{0}\right)\right), V_{0}\right) .
\end{array}\right.
$$

We then rescale the time and define $\left(\mathcal{V}_{0}, \mathcal{N}\right)(\tau, y, \theta)=\left(V_{0}, N\right)(\tau / \varepsilon, y, \theta)$. The system in $\left(\mathcal{V}_{0}, \mathcal{N}\right)$ is

$$
\left\{\begin{array}{l}
\partial_{\tau} \mathcal{V}_{0}+\frac{i}{\varepsilon^{2}} \mathcal{A}_{0} \mathcal{V}_{0}-\frac{\omega \partial_{\theta}}{\varepsilon^{2}} \mathcal{V}_{0}=2 \Pi_{0} B\left(\mathcal{V}_{0}, \mathcal{N}+J\left(\mathcal{V}_{0}, \mathcal{V}_{0}\right)\right) \\
\partial_{\tau} \mathcal{N}-\frac{\omega \partial_{\theta}}{\varepsilon^{2}} \mathcal{N}=-2 J\left(\Pi_{0} B\left(\mathcal{V}_{0}, \mathcal{N}+J\left(\mathcal{V}_{0}, \mathcal{V}_{0}\right)\right), \mathcal{V}_{0}\right)
\end{array}\right.
$$

The initial datum is

$$
\left\{\begin{array}{l}
\mathcal{V}_{0}(0, y, \theta)=\Pi_{0} V(0, y, \theta)=\Pi_{0}\left(e^{i \theta} a(y)+e^{-i \theta} \overline{a(y)}+\varepsilon a_{1}(y, \theta)+\varepsilon^{2} a_{2}(y, \theta)\right), \\
\mathcal{N}(0, y, \theta)=W_{s}(0, y, \theta)-J\left(\mathcal{V}_{0}, \mathcal{V}_{0}\right)(0, y, \theta)=\frac{1}{\varepsilon} \Pi_{s} V(0, y, \theta)-J\left(\mathcal{V}_{0}, \mathcal{V}_{0}\right)(0, y, \theta) .
\end{array}\right.
$$

Since the leading term $a(y)$ belongs to $\operatorname{ker} L(i(\omega, k))$, and $\omega \neq 0$, we have $\Pi_{s} a=0$. Then the initial datum for $\mathcal{N}$ is $O(1)$ in $H^{1}\left(\mathbb{T}_{\theta}, H_{y}^{s-2}\right)$ :

$$
\mathcal{N}(0, y, \theta)=\Pi_{s} a_{1}(y, \theta)+\varepsilon \Pi_{s} a_{2}(y, \theta)-J\left(\mathcal{V}_{0}, \mathcal{V}_{0}\right)(0, y, \theta) \in H^{1}\left(\mathbb{T}_{\theta}, H_{y}^{s-2}\right) .
$$

This gives well-posedness over diffractive times:

Proposition 3.2. The Cauchy problem (3.20)-(3.21) admits a unique solution on a time interval $\left[0, T_{2}^{*}\left[\right.\right.$, with $T_{2}^{*}>0$ independent of $\varepsilon$. Moreover, for all $T<T_{2}^{*}$, we have the estimates :

$$
\sup _{[0, T]}\left\|\left(\mathcal{V}_{0}, \mathcal{N}\right)(\tau)\right\|_{H^{1}\left(\mathbb{T}_{\theta}, H_{y}^{s-2}\right)} \leq C,
$$

where the constant $C=C(s, T)$ is independent of $\varepsilon$ and depends on $s$ through the sum of norms $\|a\|_{H_{y}^{s}}+\left\|a_{1}\right\|_{H^{1}\left(\mathbb{T}_{\theta}, H_{y}^{s-1}\right)}+\left\|a_{2}\right\|_{H^{1}\left(\mathbb{T}_{\theta}, H_{y}^{s-2}\right)}$.

Proof. The system (3.20) being symmetric hyperbolic, local-in-time well-posedness is classical. Here the bounds are uniform in $\varepsilon$. Indeed, the initial datum (3.21) is uniformly bounded with respect to $\varepsilon$ in Sobolev spaces $H^{1}\left(\mathbb{T}_{\theta}, H_{y}^{s-2}\right), s>2+1 / 2$. The $L^{2}$ estimate is uniform in $\varepsilon$ in spite of the large $1 / \varepsilon$ prefactor because the operator $-\omega \partial_{\theta}+A\left(e_{1}\right) k \partial_{\theta}+L_{0}$ is skew-adjoint, and the commutator estimates are trivial because the operator $-\omega \partial_{\theta}+A\left(e_{1}\right) k \partial_{\theta}+L_{0}$ has constant coefficients.

Back to the variable $\left(V_{0}, N\right)$, where $V_{0}$ and $N$ are introduced in (3.4) and (3.16) respectively, by the definition of $\left(\mathcal{V}_{0}, \mathcal{N}\right)$, we have that $\left(V_{0}, N\right)$ is well defined over the long time interval $\left[0, T_{2}^{*} / \varepsilon[\right.$. Back 
to the variable $V$ introduced in (1.10), this gives well-posedness over the time interval $\left[0, T_{2}^{*} / \varepsilon[\right.$ with the estimates for any $T<T_{2}^{*}$ :

$$
\sup _{[0, T / \varepsilon]}\left\|\Pi_{0} V(t)\right\|_{H^{1}\left(\mathbb{T}_{\theta}, H_{y}^{s-2}\right)} \leq C, \quad \sup _{[0, T / \varepsilon]}\left\|\Pi_{s} V(t)\right\|_{H^{1}\left(\mathbb{T}_{\theta}, H_{y}^{s-2}\right)} \leq C \varepsilon,
$$

for the same constant $C=C(s, T)$ as in Proposition 3.2

We proved the long time existence of order $O(1 / \varepsilon)$ of the solution of the Cauchy problem (1.20). Back to the original variable $v(t, y)=\left.V(t, y, \theta)\right|_{\theta=\frac{-\omega t+k y}{\varepsilon}}$, the following corollary follows immediately.

Corollary 3.3. Over the time interval $\left[0, T_{2}^{*} / \varepsilon[\right.$, the Cauchy problem (1.7)-(1.9) admits a unique solution $v$ of the form $v(t, x)=V\left(t, x, \frac{k y-\omega t}{\varepsilon}\right)$, with $V(t, x, \theta) \in L^{\infty}\left([0, T / \varepsilon]_{t}, H^{1}\left(\mathbb{T}_{\theta}, H^{s-2}\left(\mathbb{R}_{y}^{1}\right)\right)\right)$ for any $T<T_{2}^{*}$. Moreover, by Sobolev embedding, we have $|v|_{L^{\infty}} \leq C$, where $C=C(s, T)$ is given in Proposition 3.2 .

In the next section, we consider the stability of the WKB solution from Section 2 and show two convergence results.

\section{Error estimates}

For the WKB solution $V^{a}$ from Section 2, we use the projection, rescalling and normal form reduction from Section 3:

$\left(V_{0}^{a}, V_{s}^{a}\right)=\left(\Pi_{0} V^{a}, \Pi_{s} V^{a}\right), \quad W_{s}^{a}=\frac{V_{s}^{a}}{\varepsilon}, \quad N^{a}=W_{s}^{a}-J\left(V_{0}^{a}, V_{0}^{a}\right), \quad\left(\mathcal{V}_{0}^{a}, \mathcal{N}^{a}\right)(\tau)=\left(V_{0}^{a}, N^{a}\right)(\tau / \varepsilon)$.

where $J$ is defined by (3.17) and (3.18). Then the system in $\left(\mathcal{V}_{0}^{a}, \mathcal{N}^{a}\right)$ is

$$
\left\{\begin{array}{l}
\partial_{\tau} \mathcal{V}_{0}^{a}+\frac{i}{\varepsilon^{2}} \mathcal{A}_{0} \mathcal{V}_{0}^{a}-\frac{\omega \partial_{\theta}}{\varepsilon^{2}} V_{0}^{a}=2 \Pi_{0} B\left(\mathcal{V}_{0}^{a}, \mathcal{N}^{a}+J\left(\mathcal{V}_{0}^{a}, \mathcal{V}_{0}^{a}\right)\right)+\varepsilon \Pi_{0} R \\
\partial_{\tau} \mathcal{N}^{a}-\frac{\omega \partial_{\theta}}{\varepsilon^{2}} \mathcal{N}^{a}=-2 J\left(\Pi_{0} B\left(\mathcal{V}_{0}^{a}, \mathcal{N}^{a}+J\left(\mathcal{V}_{0}^{a}, \mathcal{V}_{0}^{a}\right)\right), \mathcal{V}_{0}^{a}\right)-2 \varepsilon J\left(\mathcal{V}_{0}^{a}, \Pi_{0} R\right)+\Pi_{s} R
\end{array}\right.
$$

As shown in Section 2.2, an existence time for (4.1) is $T_{1}^{*}$ (introduced in Section 2 as an existence time for (2.41) $)$. Define the perturbations

$$
\Phi(\tau, y, \theta)=\left(\mathcal{V}_{0}-\mathcal{V}_{0}^{a}\right)(\tau, y, \theta), \quad \Psi(\tau, y, \theta)=\left(\mathcal{N}-\mathcal{N}^{a}\right)(\tau, y, \theta),
$$

then the couple $(\Phi, \Psi)$ solves the following system over time interval $\left[0, T_{12}^{*}\left[\right.\right.$ with $T_{12}^{*}=\min \left\{T_{1}^{*}, T_{2}^{*}\right\}$ $\left(T_{2}^{*}\right.$ is introduced in Proposition 3.2 as an existence time of $\left(\mathcal{V}_{0}, \mathcal{N}\right)$ for $\left.(3.20)-(3.21)\right)$ :

$$
\left\{\begin{array}{l}
\partial_{\tau} \Phi+\frac{i}{\varepsilon^{2}} \mathcal{A}_{0} \Phi-\frac{\omega \partial_{\theta}}{\varepsilon^{2}} \Phi=2 \Pi_{0} B\left(\mathcal{V}_{0}^{a}, \Psi\right)+H_{0}\left(\mathcal{V}_{0}^{a}, \mathcal{N}^{a}, \Phi, \Psi\right)-\varepsilon \Pi_{0} R \\
\partial_{\tau} \Psi-\frac{\omega \partial_{\theta}}{\varepsilon^{2}} \Psi=H_{s}\left(\mathcal{V}_{0}^{a}, \mathcal{N}, \Phi, \Psi\right)+2 \varepsilon J\left(\mathcal{V}_{0}^{a}, \Pi_{0} R\right)-\Pi_{s} R
\end{array}\right.
$$

where $H_{0}$ and $H_{s}$ are defined by

$$
\left\{\begin{aligned}
H_{0}\left(\mathcal{V}_{0}^{a}, \mathcal{N}^{a}, \Phi, \Psi\right):= & 2 \Pi_{0} B\left(\Phi, \mathcal{N}^{a}+\Psi+J\left(\mathcal{V}_{0}^{a}+\Phi, \mathcal{V}_{0}^{a}+\Phi\right)\right)+2 \Pi_{0} B\left(\mathcal{V}_{0}^{a}, J\left(\Phi, \Phi+2 \mathcal{V}_{0}^{a}\right)\right) \\
H_{s}\left(\mathcal{V}_{0}^{a}, \mathcal{N}^{a}, \Phi, \Psi\right):= & 2 J\left(\Pi_{0} B\left(\mathcal{V}_{0}^{a}, \mathcal{N}^{a}+J\left(\mathcal{V}_{0}^{a}, \mathcal{V}_{0}^{a}\right)\right), \mathcal{V}_{0}^{a}\right) \\
& -2 J\left(\Pi_{0} B\left(\mathcal{V}_{0}^{a}+\Phi, \mathcal{N}^{a}+\Psi+J\left(\mathcal{V}_{0}^{a}+\Phi, \mathcal{V}_{0}^{a}+\Phi\right)\right), \mathcal{V}_{0}^{a}+\Phi\right)
\end{aligned}\right.
$$


By (2.46), the initial datum for $(\Phi, \Psi)$ is

$$
\left\{\begin{array}{l}
\Phi(0)=\left(V_{0}-V_{0}^{a}\right)(0)=-\varepsilon \Pi_{0} b-\varepsilon^{2} \Pi_{0} b_{1}, \\
\Psi(0)=\frac{1}{\varepsilon}\left(V_{s}-V_{s}^{a}\right)(0)-J\left(V_{0}, V_{0}\right)(0)+J\left(V^{a}, V^{a}\right)(0)=-\Pi_{s} b+\varepsilon b_{2},
\end{array}\right.
$$

where $b$ and $b_{1}$ are defined by (2.47) and (2.48), and $b_{2} \in H^{1}\left(\mathbb{T}_{\theta}, H_{y}^{s-2}\right)$ is defined by

$$
b_{2}=-\Pi_{s} b_{1}+J\left(b+\varepsilon b_{1}, V_{a}(0)\right)+J\left(V(0), b+\varepsilon b_{1}\right) .
$$

We give two estimates for $(\Phi, \Psi)$ in the following two sections.

\subsection{First error estimate}

Here we assume only the polarization condition $a(y) \in \operatorname{ker} L(i(\omega, k))$. Then by (4.9), we have $\Phi(0)=O(\varepsilon), \Psi(0)=O(1)$ in $H^{1}\left(\mathbb{T}_{\theta}, H_{y}^{s-2}\right)$. Then for the symmetric hyperbolic system (4.3), we have the following proposition:

Proposition 4.1. The Cauchy problem (4.3) -(4.9) admits a unique solution on time interval $\left[0, T_{3}^{*}[\right.$ with $T_{3}^{*} \geq T_{12}^{*}$, and the following estimates hold for any $T<T_{3}^{*}$ :

$$
\|\Phi\|_{L^{\infty}\left([0, T], H^{1}\left(\mathbb{T}_{\theta}, H_{y}^{s-2}\right)\right)} \leq C(\varepsilon+T), \quad\|\Psi\|_{L^{\infty}\left([0, T], H^{1}\left(\mathbb{T}_{\theta}, H_{y}^{s-2}\right)\right)} \leq C,
$$

where the constant $C=C(s, T)$ is independent of $\varepsilon$ and depends on $s$ through the sum of norms $\|a\|_{H_{y}^{s}}+\left\|a_{1}\right\|_{H^{1}\left(\mathbb{T}_{\theta}, H_{y}^{s-1}\right)}+\left\|a_{2}\right\|_{H^{1}\left(\mathbb{T}_{\theta}, H_{y}^{s-2}\right)}$.

Proof. As in the proof of Proposition 3.2, the data being bounded in $\varepsilon$, we have existence, uniqueness, and uniform bounds in $\varepsilon$ for short times with existence time $T_{3}^{*}$ independent of $\varepsilon$.

The source terms of (4.3) is $O(1)$, then we have the estimate:

$$
\|(\Phi, \Psi)\|_{L^{\infty}\left([0, T], H^{1}\left(\mathbb{T}_{\theta}, H_{y}^{s-2}\right)\right)} \leq C .
$$

By the first equation of (4.9), the initial datum $\Phi(0)=O(\varepsilon)$. The classical $H^{s}$ estimate then gives

$$
\|\Phi\|_{L^{\infty}\left([0, T], H^{1}\left(\mathbb{T}_{\theta}, H_{y}^{s-2}\right)\right)} \leq C\left(|\Phi(0)|_{L^{\infty}\left([0, T], H^{1}\left(\mathbb{T}_{\theta}, H_{y}^{s-2}\right)\right)}+\int_{0}^{T} C d t\right) \leq C(\varepsilon+T)
$$

Since we already know that $(\Phi, \Psi)$ defined by (4.2) solves (4.3)-(4.9) over time interval $\left[0, T_{12}^{*}[\right.$ where $T_{12}^{*}:=\min \left\{T_{1}^{*}, T_{2}^{*}\right\}$, we have $T_{3}^{*} \geq T_{12}^{*}$ by uniqueness.

Remark 4.2. Here we show first that the exact solution exists over long times $O(1 / \varepsilon)$ before showing that it is approximated by the WKB solution on the intersection of their intervals of existence. Indeed, the Maxwell-Bloch structure allows us to find the normal form of the nonlinear equations, in contrast to, e.g., the linear normal form reduction of [9].

Remark 4.3. For this first error estimate, we do not need the special choice of $f$ given by (2.43). The estimate (4.6), as well as the first estimate (1.24) in Theorem [1.3, hold for any regular function $f$ that satisfies the transport equation (2.38), e.g. $f=0$. (Recall, $f$ is introduced in Section 2.1 as a building block of the first corrector $V^{1}$ in WKB approximation. See (2.18)).

Back to the original time and variables, we immediately obtain the following corollary:

Corollary 4.4. For any $T<T_{12}^{*}$, we have the error estimate

$$
\left\{\begin{array}{l}
\left\|\Pi_{0}\left(V-V^{a}\right)\right\|_{L^{\infty}\left([0, T / \varepsilon], H^{1}\left(\mathbb{T}_{\theta}, H_{y}^{s-2}\right)\right)} \leq C(\varepsilon+T), \\
\left\|\Pi_{s}\left(V-V^{a}\right)\right\|_{L^{\infty}\left([0, T / \varepsilon], H^{1}\left(\mathbb{T}_{\theta}, H_{y}^{s-2}\right)\right)} \leq C \varepsilon
\end{array}\right.
$$

The constant $C=C(s, T)$ is as in Proposition 4.1 . 


\subsection{Second error estimate}

In this section, we show the stability of the WKB solution in the following sense: for some $T>0$ independent of $\varepsilon$, we have

$$
\left\|\Pi_{0}\left(V-V^{a}\right)\right\|_{L^{\infty}\left([0, T / \varepsilon], H^{1}\left(\mathbb{T}_{\theta}, H_{y}^{s-2}\right)\right)}=O(\varepsilon), \quad\left\|\Pi_{s}\left(V-V^{a}\right)\right\|_{L^{\infty}\left([0, T / \varepsilon], H^{1}\left(\mathbb{T}_{\theta}, H_{y}^{s-2}\right)\right)}=O\left(\varepsilon^{2}\right),
$$

provided the initial perturbation

$$
\left\|\Pi_{0}\left(V-V^{a}\right)(0)\right\|_{H^{1}\left(\mathbb{T}_{\theta}, H_{y}^{s-2}\right)}=O(\varepsilon), \quad\left\|\Pi_{s}\left(V-V^{a}\right)(0)\right\|_{H^{1}\left(\mathbb{T}_{\theta}, H_{y}^{s-2}\right)}=O\left(\varepsilon^{2}\right) .
$$

Here we assume that the initial corrector $a_{1}$ satisfies (2.51). Then by Lemma (2.1),

$$
\left\|\Pi_{s}\left(V-V^{a}\right)\right\|_{L^{\infty}\left([0, T / \varepsilon], H^{1}\left(\mathbb{T}_{\theta}, H_{y}^{s-2}\right)\right)}=O\left(\varepsilon^{2}\right),
$$

and the initial datum of $(\Phi, \Psi)$ (introduced in (4.2)) is

$$
\Phi(0)=-\varepsilon \Pi_{0} b-\varepsilon^{2} \Pi_{0} b_{1}=O(\varepsilon), \quad \Psi(0)=-\Pi_{s} b+\varepsilon b_{2}=\varepsilon b_{2}=O(\varepsilon) .
$$

For the remainder $R$ (introduced in (2.46) and explicitly given in (2.49)), with the choice of $f$ in (2.43), by Lemma 2.2. we have that $\Pi_{s} R=-\varepsilon R_{s}$ with $R_{s}=2 B\left(V^{1}, V^{2}\right)+\varepsilon B\left(V^{2}, V^{2}\right) \in$ $L^{\infty}\left([0, T / \varepsilon], H^{1}\left(\mathbb{T}_{\theta}, H_{y}^{s-2}\right)\right)$ for any $T<T_{1}^{*}$. We now consider the rescaled variables

$$
\Phi_{1}=\Phi / \varepsilon, \Psi_{1}=\Psi / \varepsilon
$$

then the equation in $\left(\Phi_{1}, \Psi_{1}\right)$ is

$$
\left\{\begin{array}{l}
\partial_{\tau} \Phi_{1}+\frac{i}{\varepsilon^{2}} \mathcal{A}_{0} \Phi_{1}-\frac{\omega \partial_{\theta}}{\varepsilon^{2}} \Phi_{1}=2 \Pi_{0} B\left(\mathcal{V}_{0}^{a}, \Psi_{1}\right)+\frac{1}{\varepsilon} H_{0}\left(\mathcal{V}_{0}^{a}, \mathcal{N}^{a}, \varepsilon \Phi_{1}, \varepsilon \Psi_{1}\right)-\Pi_{0} R, \\
\partial_{\tau} \Psi_{1}-\frac{\omega \partial_{\theta}}{\varepsilon^{2}} \Psi_{1}=\frac{1}{\varepsilon} H_{s}\left(\mathcal{V}_{0}^{a}, \mathcal{N}, \varepsilon \Phi_{1}, \varepsilon \Psi_{1}\right)+2 J\left(\mathcal{V}_{0}^{a}, \Pi_{0} R\right)-R_{s}
\end{array}\right.
$$

with the initial datum

$$
\Phi_{1}(0)=-\Pi_{0} b-\varepsilon \Pi_{0} b_{1}, \quad \Psi_{1}(0)=b_{2},
$$

and where $\left(H_{0}, H_{s}\right)$ are defined in (4.4). Note that by bilinearity of $B$, and pointwise bounds for the approximate solution, we have uniform bounds

$$
\frac{1}{\varepsilon}\left|H_{0}\left(\mathcal{V}_{0}^{a}, \mathcal{N}^{a}, \varepsilon \Phi, \varepsilon \Psi\right)\right| \leq C(|\Phi|+|\Psi|), \quad \frac{1}{\varepsilon}\left|H_{s}\left(\mathcal{V}_{0}^{a}, \mathcal{N}^{a}, \varepsilon \Phi, \varepsilon \Psi\right)\right| \leq C(|\Phi|+|\Psi|) .
$$

It is now classical to deduce uniform bounds for $\Phi_{1}$ and $\Psi_{1}$ in times $O(1)$ :

Proposition 4.5. With the choice of $a_{1}$ in (2.51), the Cauchy problem (4.13)-(4.14) admits a unique solution $\left(\Phi_{1}, \Psi_{1}\right)$ on $\left[0, T_{4}^{*}\left[\right.\right.$ with $T_{4}^{*}>0$ independent of $\varepsilon$, and for any $T<T_{4}^{*}$ :

$$
\left\|\Phi_{1}\right\|_{L^{\infty}\left([0, T], H^{1}\left(\mathbb{T}_{\theta}, H_{y}^{s-2}\right)\right)} \leq C, \quad\left\|\Psi_{1}\right\|_{L^{\infty}\left([0, T], H^{1}\left(\mathbb{T}_{\theta}, H_{y}^{s-2}\right)\right)} \leq C,
$$

where the constant $C=C(s, T)$ is independent of $\varepsilon$ and depends on s through the sum of norms $\|a\|_{H_{y}^{s}}+\left\|a_{1}\right\|_{H^{1}\left(\mathbb{T}_{\theta}, H_{y}^{s-1}\right)}+\left\|a_{2}\right\|_{H^{1}\left(\mathbb{T}_{\theta}, H_{y}^{s-2}\right)}$.

Remark 4.6. For this second error estimate, we do need the special choice of $f$ in (2.43). Together with the choice of initial corrector $a_{1}$ in (2.51), it allows us to rescale the solution (4.12) to obtain the desired estimates. 
Back to the original time and variables, we immediately deduce the following corollary:

Corollary 4.7. Let $T^{*}=\min \left\{T_{12}^{*}, T_{4}^{*}\right\}$, then for any $T<T^{*}$, we have the error estimates

$$
\left\|\Pi_{0}\left(V-V^{a}\right)\right\|_{L^{\infty}\left([0, T / \varepsilon], H^{1}\left(\mathbb{T}_{\theta}, H_{y}^{s-2}\right)\right)} \leq C \varepsilon, \quad\left\|\Pi_{s}\left(V-V^{a}\right)\right\|_{L^{\infty}\left([0, T / \varepsilon], H^{1}\left(\mathbb{T}_{\theta}, H_{y}^{s-2}\right)\right)} \leq C \varepsilon^{2},
$$

where the constant $C=C(s, T)$ is as in Proposition 4.5 .

This shows stability, that is, the WKB approximate profile stays close to the exact profile over its existence time with an error estimate that is comparable to the initial error.

\subsection{Proof of Theorem 1.3}

We now sum up and prove Theorem 1.3 .

First, by Corollary 3.3, the function $v(t, y)=\left.V(t, y, \theta)\right|_{\theta=\frac{k y-\omega t}{\varepsilon}}$ solves (1.7)-(1.9) over time interval $\left[0, T^{*}[\right.$, and this gives the first result of Theorem 1.3$]$

Second, by the Corollary 4.4 and Sovolev embedding $H^{1}\left(\mathbb{T}_{\theta}, H_{y}^{s-2}\right) \subset L^{\infty}\left(\mathbb{T}_{\theta} \times \mathbb{R}_{y}\right)$, the error estimates (1.24) and (1.26) of Theorem 1.3 follow immediately.

Finally, by the Corollary 4.7 and Sovolev embedding $H^{1}\left(\mathbb{T}_{\theta}, H_{y}^{s-2}\right) \subset L^{\infty}\left(\mathbb{T}_{\theta} \times \mathbb{R}_{y}\right)$, we have the second error estimate (1.25). This shows stability of the approximate solution.

Acknowledgment. I warmly thank Eric Dumas and Isabelle Gallagher for stimulating discussions. I particularly thank my advisor Benjamin Texier for his fruitful remarks and for suggesting this interesting subject.

\section{References}

[1] T. Colin, Rigorous derivation of the nonlinear Schrödinger equation and DaveyStewartson systems from quadratic hyperbolic systems, Asymptotic Analysis (2002), 69-91.

[2] T. Colin, D. Lannes, Justification of and long-wave correction to Davey-Stewartson systems from quadratic hyperbolic systems, Discrete and Continuous Dynamical Systems, vol. 11, n. 1 (2004), 83-100.

[3] J.-L. Joly, G. Métivier, J. Rauch, Dispersive nonlinear geometric optics with rectification, Indiana U. Math. J., vol. 47 (1998), 1167-1241.

[4] J.-L. Joly, G. Métivier, J. Rauch, Transparent nonlinear geometric optics and Maxwell-Bloch equations, J. Diff. Eq., vol. 166 (2000), 175-250.

[5] D. Lannes, Dispersive effects for nonlinear geometrical optics with rectification, Asymptotic Analysis, vol. 18 (1998), 111-146.

[6] D. Lannes, Secular growth estimates for hyperbolic systems, J. Diff. Eq.,vol. 190 (2003), 466-503.

[7] H. Leblond, Electromagnetic waves in ferromagnets: a Davey-Stewartson-type model, J. Phys. A: Math. Gen., vol. 32, (1999), 7907-7932. 
[8] B. Texier, The short wave limit for nonlinear, symmetric hyperbolic systems, Adv. Diff. Eq. 9 (2004), no. 1, 1-52.

[9] B. Texier, Derivation of the Zakharov equations, Arch. Rational Mech. Anal vol. 184 (2007), 121-183. 